\title{
Expression of Neural RNA-Binding Proteins in the Postnatal CNS: Implications of Their Roles in Neuronal and Glial Cell Development
}

\author{
Shin-ichi Sakakibara ${ }^{1}$ and Hideyuki Okano ${ }^{1,2}$ \\ ${ }^{1}$ Department of Neuroanatomy, Biomedical Research Center, Osaka University Medical School, Suita, Osaka 565, Japan, \\ and ${ }^{2}$ Core Research for Evolutional Science and Technology (CREST), Japan Science and Technology Corporation, \\ Shinagawa, Tokyo 140, Japan
}

\begin{abstract}
There is an increasing interest in the role of RNA-binding proteins during neural development. Mouse-Musashi-1 (m-Msi-1) is a mouse neural RNA-binding protein with sequence similarity to Drosophila musashi $(d-m s i)$, which is essential for neural development. m-Msi-1 is highly enriched in neural precursor cells that are capable of generating both neurons and glia during embryonic CNS development. The present study characterized $\mathrm{m}$-Msi-1-expressing cells in the postnatal and adult CNS. Postnatally, m-Msi-1 was expressed in proliferative neuronal precursors in the external granule cell layer of the cerebellum and in the anterior corner of the subventricular zone of the lateral ventricles. In gliogenesis, the persistent expression of m-Msi-1 was observed in cells of the astrocyte lineage ranging from proliferative glial precursors in the subventricular
\end{abstract}

zone (SVZ) to differentiated astrocytes in the parenchyma. In addition, we showed that m-Msi-1 was still expressed in proliferating cells in the adult SVZ, which may contain neural precursor or stem cells. Another neural RNA-binding protein $\mathrm{Hu}$ (the mammalian homolog of a Drosophila neuronal RNAbinding protein Elav) was present in postmitotic neurons throughout the development of the CNS, and its pattern of expression was compared with that of $\mathrm{m}-\mathrm{Msi}-1$. These observations imply that these two RNA-binding proteins may be involved in the development of neurons and glia by regulating gene expression at the post-transcriptional level.

Key words: RNA-binding proteins; postnatal CNS; mouseMusashi-1 (m-Msi-1); Hu; Drosophila musashi; neuronal and glial precursor cells; astrocyte lineage
A number of transcription factors that function in the proliferation and differentiation of neural precursor cells have been identified. However, the recent discovery of neural-specific RNAbinding proteins raises the possibility that the development of neural cells from the precursors may also be regulated at the post-transcriptional level. We believe that at least two gene families are represented by the neural RNA-binding proteins found in both invertebrates and vertebrates (Okano, 1995; Sakakibara et al., 1996). One, the Elav family, includes Drosophila elav genes (Yao et al., 1993) and their mammalian homologs, the $H u$ genes (Szabo et al., 1991). Members of this family share extensive sequence similarity with one another, and they seem to be expressed mainly in postmitotic neurons (Okano and Darnell, 1997). The other Msi family is composed of Drosophila musashi (Nakamura et al., 1994), Xenopus laevis nrp-1 (Richter et al., 1990), and their mouse homolog mouse-musashi-1 (m-msi-1) (Sakakibara et al., 1996). d-Msi is required for the two successive asymmetric cell divisions of sensory organ precursors (Nakamura et al., 1994). In contrast to the Elav family, the members of the Msi family are expressed in the neural precursor cells at least during embryonic CNS development (Sakakibara et al., 1996). Although the molecular functions and the in vivo RNA targets of

Received May 14, 1997; revised Aug. 18, 1997; accepted Aug. 19, 1997.

This work was supported by grants from the Japanese Ministry of Education, Science and Culture (S.S., H.O.), and by Core Research for Evolutional Science and Technology (CREST), Japan Science and Technology Corporation. S.S. is a research fellow of the Japan Society for the Promotion of Science. We thank Drs.

Miyuki Yamamoto and Masato Nakafuku for their critical reading of this manuscript.

Correspondence should be addressed to Dr. Hideyuki Okano, Department of Neuroanatomy, Biomedical Research Center, Osaka University, Suita, Osaka 565, Japan.

Copyright (C) 1997 Society for Neuroscience $\quad 0270-6474 / 97 / 178300-13 \$ 05.00 / 0$
Msi and Elav families remain largely unknown, these different expression patterns suggest that the Msi and Elav families may play distinct roles in the development and maintenance of neural cells.

During CNS development, neurons and glial cells are thought to be generated from common neural precursor cells (multipotent CNS stem cells) (Davis and Temple, 1994). Our previous study has suggested that m-Msi-1 is expressed predominantly in proliferating multipotent neural precursor cells that may give rise to neurons and/or glia, but is not expressed in newly generated postmitotic neurons (Sakakibara et al., 1996). The expression of m-Msi-1 is largely downregulated with the successive progression of neurogenesis. However, a considerable amount of m-Msi-1 transcripts is present in the brain during postnatal development, and low level expression persists into adulthood (Sakakibara et al., 1996). Recent studies have indicated the presence of neural precursor cells in postnatal neural development. To further study the role of m-Msi-1 in neural precursor cells in the postnatal and adult CNS, the spatiotemporal distribution of m-Msi-1-expressing cells was examined. The m-Msi-1 protein can be detected in proliferating neuronal and/or glial precursor cells in the SVZ and in cells of the astrocyte lineage in the postnatal and adult CNS. These observations suggest also that m-Msi-1 may be an excellent marker for protoplasmic astrocytes.

\section{MATERIALS AND METHODS}

Animals and tissue preparation. ICR mice and Wistar rats used throughout the experiments were purchased from Charles River Japan Inc. The day of birth was designated as postnatal day (P) 0 . Pups and adults (anesthetized by ether inhalation) were perfused through the left ventricle with $4 \%$ paraformaldehyde in $0.1 \mathrm{M} \mathrm{PBS,} \mathrm{pH} 7.4$, and then the brains were dissected and post-fixed overnight at $4^{\circ} \mathrm{C}$ in the same fixative. For paraffin sectioning, 
tissue blocks were rinsed twice with PBS for 30 min each time and dehydrated through a series of increasing concentrations of ethanol. After dehydration, they were cleared with chloroform and xylene and then embedded in paraffin (Tissue Prep, Fisher, Pittsburgh, PA) at $62^{\circ} \mathrm{C}$. Sections (4 $\mu \mathrm{m})$ were cut on a microtome and mounted on gelatin/chrome-coated slides. Paraffin sections were used for hematoxylin-eosin and immunohistochemical staining with a single primary antibody. For cryosectioning sample blocks rinsed with PBS were cryoprotected in $30 \%$ sucrose in PBS overnight at $4^{\circ} \mathrm{C}$, embedded in O.C.T. compound (Tissue Tek, Miles, Elkhart, IN), and frozen on dry ice. Cryostat sections $(12 \mu \mathrm{m})$ were cut and affixed to glass slides precoated with gelatin/chrome. These sections were used for double-immunostaining.

Immunohistochemical analysis. The following primary antibodies were used: m-Msi-1 (affinity-purified rabbit polyclonal antibody, used at 1:500 dilution) (Sakakibara et al., 1996); Hu proteins (mouse monoclonal $\mathrm{IgG} 2 \mathrm{~B}$, clone $16 \mathrm{~A} 11$, which binds to $\mathrm{Hu}$ proteins including $\mathrm{HuD}, \mathrm{HuC}$, and HelN-1, supplied by Dr. M. F. Marusich, University of Oregon; used at 1:500 dilution) (Marusich et al., 1994); nestin (mouse monoclonal IgG, clone RE6-96 supplied by Dr. M. Ogawa, Kochi Medical School, Japan); ascitic fluid used at 1:1000 dilution (Miyata and Ogawa, 1994); proliferating cell nuclear antigen (PCNA) (mouse monoclonal IgG1, used at 1:200 dilution; Novocastra Laboratories); glial fibrillary acidic protein (GFAP) (mouse monoclonal IgG1, clone G-A-5, used at 1:400 dilution) (Sigma, St. Louis, MO); 2', 3'-cyclic nucleotide-3'-phosphohydrolase (CNPase) (mouse monoclonal IgG1, clone 11-5B, used at 1:100 dilution) (Sigma); bromodeoxyuridine (BrdU) (mouse monoclonal IgG1, clone BU-33, used at 1:5000 dilution) (Sigma); MacI (mouse monoclonal IgG1, used at 1:100 dilution) (Boehringer Mannheim, Indianapolis, IN), platelet-derived growth factor $\alpha$-receptor (PDGF $\alpha$-R) (rat monoclonal IgG, a kind gift from Dr. S.-I. Nishikawa, Kyoto University, Japan; used at 1:5000 dilution) (Takakura et al., 1996), and NG2 (mouse monoclonal IgG1, a kind gift from Dr. W. D. Richardson, University College London, UK; used at 1:100 dilution) (Stallcup and Beasley, 1987). Secondary and tertiary reagents were obtained commercially from Vector Laboratories (Burlingame, CA) or Jackson ImmunoResearch (West Grove, PA). The specificity of the polyclonal antibody to m-Msi-1 was confirmed by the immunoblot analysis of the $m-m s i-1$ knock-out mice (our unpublished results). Immunolabeling with a single primary antibody was performed with an avidin-biotin-peroxidase technique. Briefly, dewaxed and rehydrated serial paraffin sections were quenched for endogenous peroxidase activity by treating with $0.3 \%$ hydrogen peroxide in methanol for $30 \mathrm{~min}$ at room temperature, blocked for $1 \mathrm{hr}$ in $10 \%$ normal goat serum and $0.1 \%$ Triton X-100 in PBS, and then incubated with a primary antibody diluted in the same blocking solution overnight at $4^{\circ} \mathrm{C}$. Sections were placed in an appropriate biotinylated secondary antibody diluted with the blocking solution (biotinylated goat anti-mouse, rat, or rabbit $\mathrm{IgG}$; Jackson) at 1:750 for $1 \mathrm{hr}$, followed by incubation with avidin-conjugated horseradish peroxidase (HRP at 1: 1000) (Vector), and HRP was visualized using $0.5 \mathrm{mg} / \mathrm{ml}$ of diaminobenzidine (DAB) and $0.03 \% \mathrm{H}_{2} \mathrm{O}_{2}$. Each step was followed by four washes in PBS. Double indirect immunostaining was performed on the frozen sections; the cells were permeabilized in $0.1 \%$ Triton X-100 in PBS for $30 \mathrm{~min}$ and blocked as above. Polyclonal antibody to m-Msi-1 was used in combination with antibodies against $\mathrm{Hu}$, GFAP, CNPase, BrdU, MacI, PDGF $\alpha$-R, NG2, or nestin. After four washes with PBS, bound antibodies were visualized by incubation with fluorochrome-conjugated secondary antibodies: Cy-3-, or fluorescein-isothiocyanate (FITC)labeled goat anti-mouse IgG, FITC-labeled goat anti-rabbit IgG, and Texas Red-labeled goat anti-rat IgG (used at 1:200 dilution with $0.1 \%$ Triton X-100 in PBS; Jackson). After another four washes, the sections were mounted and examined with a Leitz DMRD microscope equipped with L4, ND, N2.1, and TX (Leica Inc., Buffalo, NY) epifluorescent filters. Laser scanning confocal microscopy was performed with a Zeiss LSM410 cofocal imaging system.

$B r d U$ incorporation in vivo. To label the dividing cells in the postnatal SVZ, P7 mice were given two intraperitoneal injections, $1.5 \mathrm{hr}$ apart, of $100 \mathrm{mg} / \mathrm{kg}$ of body weight of BrdU (Sigma) dissolved in PBS, and then were killed by perfusion $1.5 \mathrm{hr}$ after the second injection. In adult animals (3 months old), to label the entire population of proliferating subependymal cells, which have a cell cycle time of $\sim 12.7 \mathrm{hr}$ in mouse (Morshead and van der Kooy, 1992), BrdU (75 mg/kg of body weight) was injected intraperitoneally every $2 \mathrm{hr}$ for $12 \mathrm{hr}$, and the animals were killed by perfusion $0.5 \mathrm{hr}$ after the last injection. The BrdU-injected brains were removed and post-fixed overnight at $4^{\circ} \mathrm{C}$, and serial frozen sections were prepared in the coronal plane as described above. For immunostaining with anti-BrdU antibody, sections were incubated in $1 \mathrm{~N}$ $\mathrm{HCl}$ for $30 \mathrm{~min}$ at $60^{\circ} \mathrm{C}$ to denature the DNA, followed by $0.1 \mathrm{M}$ sodium borate, $\mathrm{pH} 8.5$, for $10 \mathrm{~min}$. After three washes with PBS, indirect double-immunolabeling for BrdU and m-Msi-1 was performed as described above.

Surgical procedures for brain lesions. Adult ICR mice, 3 months old, were anesthetized with Nembutal injections $(10 \mathrm{mg} / \mathrm{kg}$ ) (Abbott Laboratories, North Chicago, IL), and a midline skin incision was made. A unilateral linear craniectomy was performed on the skull by using a drill $3 \mathrm{~mm}$ left of the midline. The wound was made in the left cerebral hemisphere; a 27 gauge stainless steel needle was placed $3 \mathrm{~mm}$ lateral to the sagittal fissure and $2 \mathrm{~mm}$ anterior to the lambda line, and inserted through the pia to a depth of $1.5 \mathrm{~mm}$. The brain was cut sagittally $4 \mathrm{~mm}$ anterior to the lambda line. The creviced skull was filled with Bonewax, and the wound was closed with sutures. These lesioned animals showed no obvious behavioral or motor deficits. At $24 \mathrm{hr}$ and $4 \mathrm{~d}$ after surgery, the lesioned animals were given two intraperitoneal injections of BrdU (100 mg/kg of body weight) (Sigma) over a $2 \mathrm{hr}$ period and were again anesthetized and then killed by perfusion $2 \mathrm{hr}$ after the second injection. The brains were removed, and serial frozen sections were cut in the coronal plane at the level of the wound and processed for doubleimmunofluorescent staining as described above.

\section{RESULTS}

\section{m-Msi-1 expression in the SVZ of the forebrain}

In the present study we characterized postnatal expression of m-Msi-1 in the CNS (Table 1). Consistent with the previous Northern blot analysis (Sakakibara et al., 1996), the immunoblot analysis indicated that the expression of m-Msi-1 remained in CNS during the postnatal development, albeit at a lower level than that observed in the embryonic CNS (data not shown). An immunohistochemical analysis was performed to examine the spatiotemporal pattern of m-Msi-1 expression in the postnatal brain. We first examined m-Msi-1 expression in the early postnatal forebrain, which contains the SVZ. In the P3 forebrain, a significant level of m-Msi-1 expression was observed in the tightly packed round cells in the SVZ, which was composed of PCNApositive proliferating cells (Fig. 1D,E) (Galand and Degraef, 1989), near the anterior part of the lateral ventricle (SVZa), and was also detected in the migratory pathway toward the olfactory bulb (Fig. 1B). This pattern of expression corresponded to the well described pathway of neuronal precursor cells, which lack radial glial fibers, to the olfactory bulb, where they differentiate into the interneurons of the granule cell layer and the glomerular layer (Luskin, 1993). Therefore, m-Msi-1 is probably expressed in neuronal precursor cells and immature interneurons migrating into the olfactory bulb. In addition to the SVZa, m-Msi-1 was also expressed uniformly in the posterior regions of the SVZ, which also contain PCNA-positive proliferating cells (Figs. $1 B, 6 A, B$ ), corresponding to the region that exclusively contains glial precursors (Privat, 1975). Taken together, these results suggest that $\mathrm{m}$-Msi-1 expression is associated with gliogenesis as well as neurogenesis in the early postnatal forebrain.

To determine whether m-Msi-1 is expressed in proliferating glial precursor cells within the developing SVZ, we pulse-labeled the SVZ of P7 mice with BrdU for $3 \mathrm{hr}$ before the mice were killed, and prepared coronal sections. With combined m-Msi-1 and BrdU immunostaining, it was possible to distinguish the expression of m-Msi-1 in dividing cells from that in postmitotic cells throughout the labeling period. Consequently, the majority of cells expressing m-Msi-1 were confined to SVZ regions and ependymal cells surrounding the lateral ventricle in a pattern similar to that of BrdU incorporation (Fig. $2 A, B$ ). In particular, a large number of m-Msi-1 and BrdU double-positive dividing cells were detected in the dorsolateral area (Fig. 2A,B, insets), where precursors for astrocytes and oligodendrocytes are more enriched 


\begin{tabular}{|c|c|c|c|}
\hline Region & P3-P7 & Adult & \\
\hline $\begin{array}{l}\text { SVZ and ventricular zone (lateral, } \\
\text { third and fourth ventricles) }\end{array}$ & +++ & +++ & $\begin{array}{l}\text { SVZ cells (subependymal cells) } \\
\text { Ependymal cells }\end{array}$ \\
\hline \multicolumn{4}{|l|}{ White matter tracts } \\
\hline Corpus callosum & ++ & ++ & Astrocytes \\
\hline Cerebellar folia white matter & ++ & + & Astrocytes \\
\hline \multicolumn{4}{|l|}{ Cerebral cortex } \\
\hline Layer I & + & ++ & Astrocytes (fibrous?) \\
\hline Layers II-V I & + & ++ & Astrocytes (protoplasmic?) \\
\hline \multicolumn{4}{|l|}{ Hippocampus } \\
\hline CA1 & - & - & \\
\hline CA2 & - & - & \\
\hline CA3 & - & - & \\
\hline Dentate gyrus & + & + & Astrocytes (subgranular layer) \\
\hline Hilus & + & + & Astrocytes \\
\hline Stratum radiatum & + & + & Astrocytes \\
\hline \multicolumn{4}{|l|}{ Basal nuclei } \\
\hline Caudate-putamen & ++ & + & Astrocytes \\
\hline Globus pallidus & ++ & + & Astrocytes \\
\hline \multicolumn{4}{|l|}{ Colliculi } \\
\hline Inferior & + & + & Astrocytes \\
\hline Superior & + & + & Astrocytes \\
\hline \multicolumn{4}{|l|}{ Cerebellum } \\
\hline External granular cell layer & ++ & - & \\
\hline Molecular layer & ++ & + & Stellate cells \\
\hline Purkinje cell layer & ++ & + & Bergmann glia \\
\hline Internal granular cell layer & + & + & Astrocytes \\
\hline
\end{tabular}

The immunoreactivities are assessed as intense, +++ ; moderate, ++ ; weak, + ; or not detectable, - .

than in other areas such as the medial and dorsal areas of the SVZ (Levison and Goldman, 1993; Levison et al., 1993; Zerlin et al., 1995). These observations strongly suggested that m-Msi-1 expression occurs in the proliferating glial precursors located within the developing SVZ. Cells immunoreactive for m-Msi-1 within the SVZ seemed to be tightly packed and to have small round cell bodies (Figs. $2 A, 6 A$ ), with a morphology similar to those described previously for glial precursor cells (Levison and Goldman, 1993; Levison et al., 1993; Zerlin et al., 1995).

To characterize the m-Msi-1-expressing cells in this region, double-label indirect immunostaining was performed with antibodies to m-Msi-1 and to a cell-type specific marker protein, including the $\mathrm{Hu}$ proteins $\mathrm{HuD}$ (Szabo et al., 1991), HuC (Liu et al., 1995), and HelN-1 (Levine et al., 1993), which label immature and differentiated neurons, CNPase for myelinating and nonmyelinating differentiated oligodendrocytes (Sprinkle, 1989), and GFAP for astrocytes. GFAP is an intermediate filament that is specifically expressed in mature astrocytes (Bignami and Dahl, 1974). Hu-positive immature neurons and CNPase-positive oligodendrocytes were visible within the dorsolateral region of the SVZ at P7, but we observed no m-Msi-1 immunoreactivity in these cells (Fig. $3 A-D$, arrows). GFAP immunoreactivity was not detected in the dorsolateral corner of the SVZ (Fig. $3 F$ ). These observations suggested that m-Msi-1 is not expressed in these differentiated cells but is confined to immature and proliferating neuronal and glial precursors in the developing SVZ.

As gliogenesis proceeds in postnatal development, the SVZ becomes thinner. However, it persists throughout adulthood as a mitotically active layer known as the subependyma (Smart, 1961; Lewis, 1968; Privat and Leblond, 1972). Recent in vivo and in vitro studies have suggested that the subependyma of the adult mammalian forebrain may be a source of neural stem cells, which may proliferate and differentiate into both neurons and glia (Lois and Alvarez-Buylla, 1993, 1994; Morshead et al., 1994; Craig et al., 1996; Gritti et al., 1996; Johe et al., 1996). To test whether m-Msi-1 is expressed in neural stem cells including the constitutively proliferating population in the adult subependyma, we pulse-labeled adult mice with BrdU for $12.5 \mathrm{hr}$ before they were killed, resulting in the labeling of the entire proliferating population of subependymal cells, the constitutively proliferating cells known to have a cell-cycle time of $\sim 12.7 \mathrm{hr}$ in mouse (Morshead and van der Kooy, 1992). The BrdU-incorporated cells were mostly confined to the subependymal region surrounding the SVZ but were not detected in the parenchyma surrounding the subependyma (Fig. 2D). In particular, the dorsolateral corner of the subependyma bordered by the striatum and overlying corpus callosum contained many proliferating cells that incorporated BrdU (Fig. 2D). These distributions of constitutively proliferating cells were consistent with the results from previous studies (Morshead et al., 1994; Gates et al., 1995). Similarly, many m-Msi-1positive cells were distributed within most regions of the subependyma and ependyma (Fig. $2 C$ ). Double-immunofluorescence revealed that the majority of the BrdU-positive cells in the subependyma were also immunoreactive for m-Msi-1 (Fig. 2C,D, arrowheads). The double-positive cells predominantly observed in the dorsolateral region of the subependyma were tightly packed, small round cells, similar to the dividing cells found in the developing SVZ described above. In addition, some populations of cells were only labeled with m-Msi-1 within the ependyma and subependyma (Fig. 2C,D, arrows). These m-Msi-1-positive and 


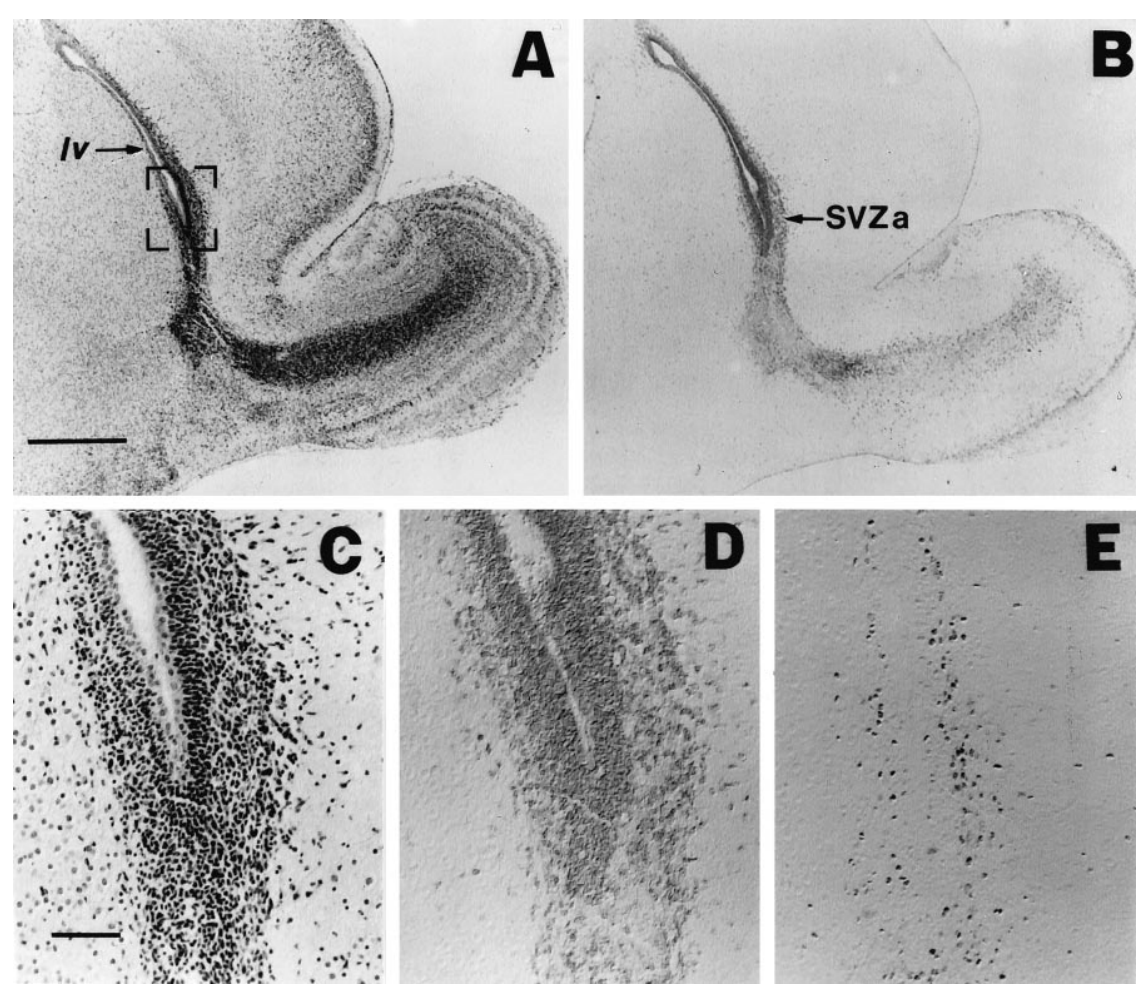

Figure 1. m-Msi-1 expression in P3 forebrain. Serial sagittal sections of the forebrain at P3 with hematoxylin-eosin staining $(A, C)$, immunolocalizations of $\mathrm{m}$ Msi-1 $(B, D)$, and PCNA $(E)$. Anterior is to the right and dorsal is up. $C-E$, Higher magnifications of the anterior corner of the lateral ventricle $(S V Z a)$ shown in the bracketed portion of $A$. m-Msi-1 immunostaining was observed in PCNA-positive proliferating cells in SVZa. Note that m-Msi-1 expression was also detected in the posterior SVZ in addition to the migratory route of neuronal precursor cells from SVZa into the olfactory bulb described previously (Luskin, 1993). Scale bars: $A, B, 500 \mu \mathrm{m} ; C-E, 50 \mu \mathrm{m}$. $S V Z a$, Anterior region of the SVZ; $l v$, lateral ventricle.
BrdU-negative cells may represent the slowly dividing stem cells, which are shown to have a cell-cycle time of $>12.7 \mathrm{hr}$ and replenish the constitutively proliferating neural precursors in the subependyma (Morshead and van der Kooy, 1992; Morshead et al., 1994). The distribution of m-Msi-1-positive cells in the adult subependyma also supported the idea that m-Msi- 1 is expressed in proliferative neuronal and/or glial precursor cells.

\section{m-Msi-1 expression in the cerebrum}

In the postnatal cerebrum, immature neurons and glial cells, which had originated from the embryonic ventricular zone or the postnatal SVZ, respectively, migrate into appropriate layers through formative white matter, including the corpus callosum that lies adjacent to the SVZ. The completion of cerebral cortex stratification occurs at around P6 in mouse. As described above, it was obvious that there were a large number of m-Msi-1-positive and densely packed cells localized within the SVZ from early postnatal stages into adulthood. However, some populations of m-Msi-1-expressing cells were also found in other parenchymal regions of the cerebrum into adulthood, including the cerebral cortex, corpus callosum, and striatum, whereas they showed a lower immunoreactivity and a sparser distribution than those found in the SVZ.

\section{The white matter}

In formative white matter, including the corpus callosum in the early postnatal period, cells immunoreactive for m-Msi-1 seemed to be distributed sparsely and to contain populations that had unior multipolar processes (Figs. $3 G, I, K, 6 A$ ) rather than the immature round cells observed in the developing SVZ (Figs. 2A, 6A). These m-Msi-1-positive cells were similar to those of early differentiating astrocytes derived from the SVZ that migrate to the cortex, subcortical white matter, and striatum, as described previously (Zerlin et al., 1995). As early as P7, GFAP was evident in the corpus callosum, where some cells bearing multiple processes or short branches were stained with both m-Msi-1 and GFAP (Fig. $3 K, L$, arrowheads). m-Msi-1 is therefore also expressed in astrocytes, including GFAP-negative immature astrocytes, in the developing white matter.

At this early stage, although the number of cells positive for CNPase or Hu was low in this region, neither of the antigens were colocalized with m-Msi-1 protein (Fig. 3G-J, arrows), indicating that astrocytes retain expression of $\mathrm{m}-\mathrm{Msi}-1$, whereas it is not expressed in differentiated or immature neurons and oligodendrocytes. Some antigenic markers have been used for analysis of the sequential differentiation of oligodendrocytes from dividing precursors, including O-2A progenitor cells, into quiescent oligodendrocytes in vitro and in vivo (Hardy and Reynolds, 1991; Nishiyama et al., 1996). PDGF $\alpha$-R is expressed in the developing and adult rodent $\mathrm{CNS}$ by the O-2A oligodendrocyte progenitors and preoligodendrocytes (Pringle et al., 1992; Ellison and de Vellis, 1994; Nishiyama et al., 1996). Similarly, NG2, a core membrane protein associated with chondroitin sulfate proteoglycan, is also expressed in mitotic O-2A progenitor cells localized in the gray and white matter of perinatal to adult rat brain and disappears as these cells differentiate into oligodendrocytes (Stallcup and Beasley, 1987; Nishiyama et al., 1996). Although the majority of NG2-positive cells also express PDGF $\alpha$-R, a recent detailed immunohistochemical comparison of the expression of PDGF $\alpha$-R and NG2 throughout CNS development demonstrated that the small population of the PDGF $\alpha$-R-positive NG2-negative cells lies in the SVZ through the first postnatal week (Nishiyama et al., 1996). These NG2negative, PDGF $\alpha$-R-positive cells residing in the SVZ were proposed to be the earliest form of O-2A progenitor cells, which divide in the SVZ to give rise to slightly more differentiated cells of the oligodendrocyte lineage expressing both NG2 and PDGF $\alpha$-R in the nascent parenchyma, such as the corpus callosum and the cortex. To determine whether m-Msi-1 is expressed in the precursor cells committed to become oligodendrocytes $(\mathrm{O}-2 \mathrm{~A}$ progenitor 
Figure 2. m-Msi-1 expression in proliferating cells residing in the postnatal and adult SVZ. To label the entire constitutively proliferating population surrounding the lateral ventricles, $\mathrm{P} 7$ and adult mice received 3 and $12.5 \mathrm{hr}$ of BrdU injections, respectively. Double-immunofluorescent labeling of coronal sections through the SVZ surrounding the lateral ventricle in $\mathrm{P} 7$ forebrain $(A$, $B)$ and adult forebrain $(C, D)$ with antibodies to m-Msi-1 ( $A, C$; FITC) and BrdU (B, $D$; Cy3). Lateral is to the right and dorsal is up. Insets in $A$ and $B$, Higher magnifications of the dorsolateral corner of the P7 SVZ. Many dividing, small, densely packed cells that are brightly immunostained with both $\mathrm{m}-\mathrm{Msi}-1$ and BrdU are observed in the postnatal developing SVZ (arrowheads in insets in $A$ and $B$ ). Similarly, a considerable number of m-Msi-1- and BrdU-positive dividing cells are also present in the adult subependyma (arrowheads in $C$ and $D$ ), although it is obvious that there is a subpopulation of m-Msi-1-positive but BrdUnegative cells (arrows in $C$ and $D$ ). Scale bars: $A, B, 36 \mu \mathrm{m}$; insets, $C$ and $D, 18 \mu \mathrm{m}$. Asterisks, Lateral ventricle; $c c$, corpus callosum; str, striatum.
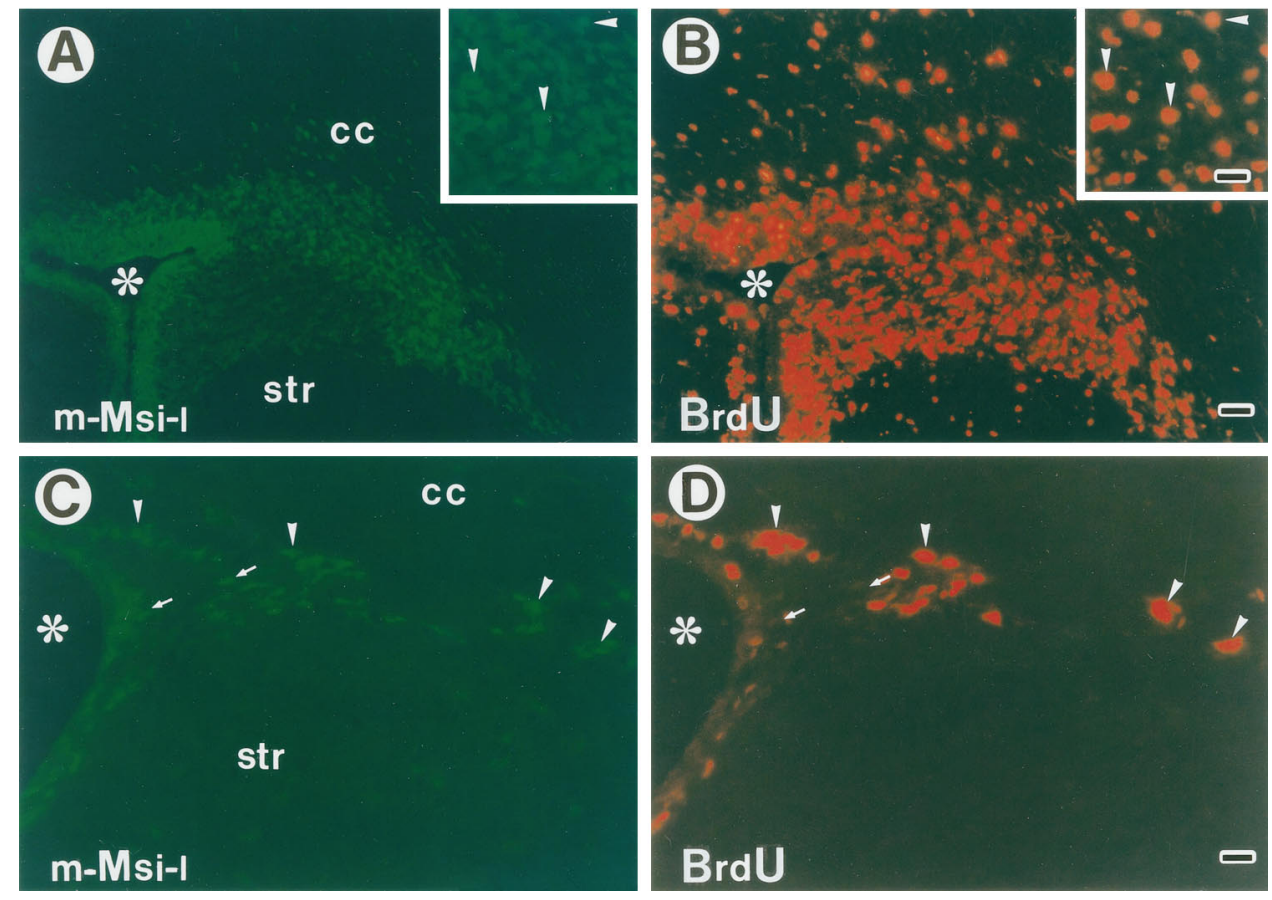

Figure 3. Cell type of m-Msi-1-positive cells in the developing SVZ and the corpus callosum. Double-labeling of coronal sections just dorsolateral to the region of the P7 SVZ $(A-F)$ and the P7 corpus callosum $(G-L)$ with anti-m-Msi-1 antibody $(A, C, E$, $G, I$, and $K$; FITC), anti-Hu ( $B, H$; Cy 3 ), anti-CNPase $(D, J ; C y 3)$, and anti-GFAP $(F, L ; \mathrm{Cy} 3)$. The bar in $E$ represents $18 \mu \mathrm{m}$. Antibodies to CNPase and Hu label populations that are distinct from m-Msi-1positive cells in both the SVZ and the corpus callosum. The arrows point to the $\mathrm{Hu}-$ positive but m-Msi-1-negative cells $(A, B$, $G, H$ ), and CNPase-positive but m-Msi-1negative cells $(C, D, I, J)$. Although GFAP immunoreactivity is not detected in the SVZ, a few m-Msi-1- and GFAP-positive cells that have multiple processes or short branched processes are now present in the developing corpus callosum (arrowheads in $K$ and $L$ ) among the more numerous m-Msi1-positive cells.

\section{SVZ}
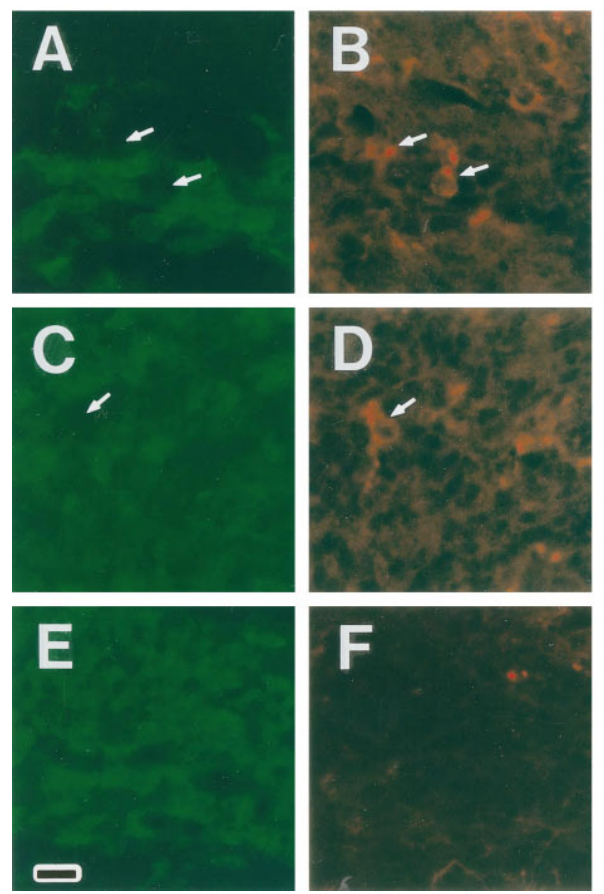
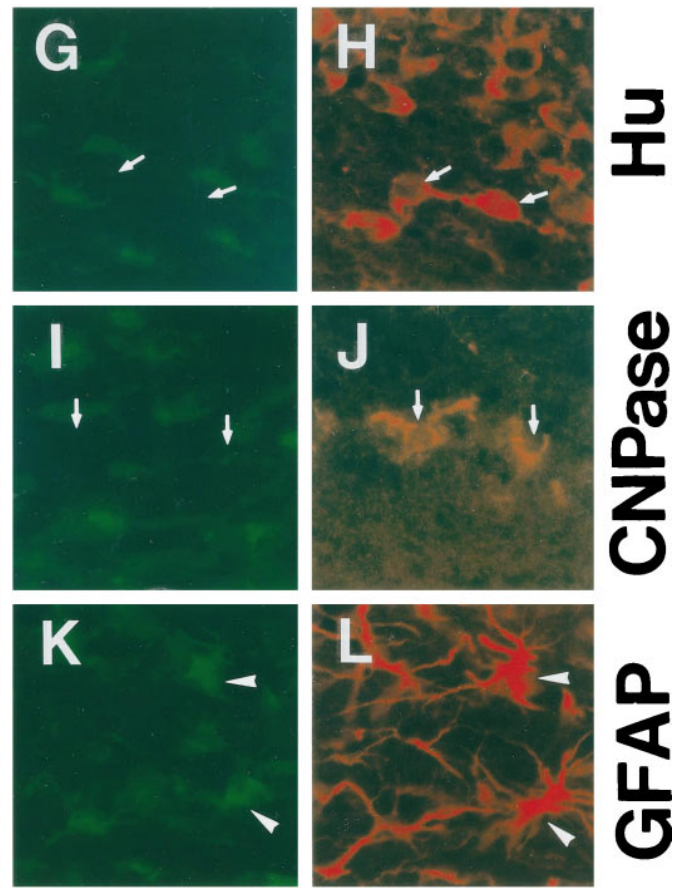

cells), double-immunofluorescent labeling with antibody to PDGF $\alpha$-R or NG2 was performed with sections prepared from the P2 mouse or the P7 rat forebrain, respectively. Double-labeling for m-Msi-1 and PDGF $\alpha$-R, however, demonstrated that these PDGF $\alpha$-R-positive cells did not overlap with the population of cells reactive for m-Msi-1 within both the SVZ and the adjacent parenchyma (Fig. 4). In addition, in the developing rat corpus callosum (P7), there were a considerable number of sparsely distributed NG2-positive cells (Fig. 5), whereas we could not detect any cells reactive for NG2 within the SVZ (data not shown), where $\mathrm{m}$-Msi-1-positive cells were condensed. Double-labeling for $\mathrm{m}$ Msi-1 and NG2 failed to reveal any double-positive cells in the corpus callosum. Cells immunoreactive for m-Msi-1 in the corpus callosum (Fig. 5, arrows) seemed to possess more rounded cell bodies and slightly shorter processes than those of NG2-positive cells (Fig. 5, arrowheads). The optic nerve of the early postnatal rat brain is also known to contain a large number of proliferating and migrating O-2A progenitor cells (Miller et al., 1985; Small et al., 

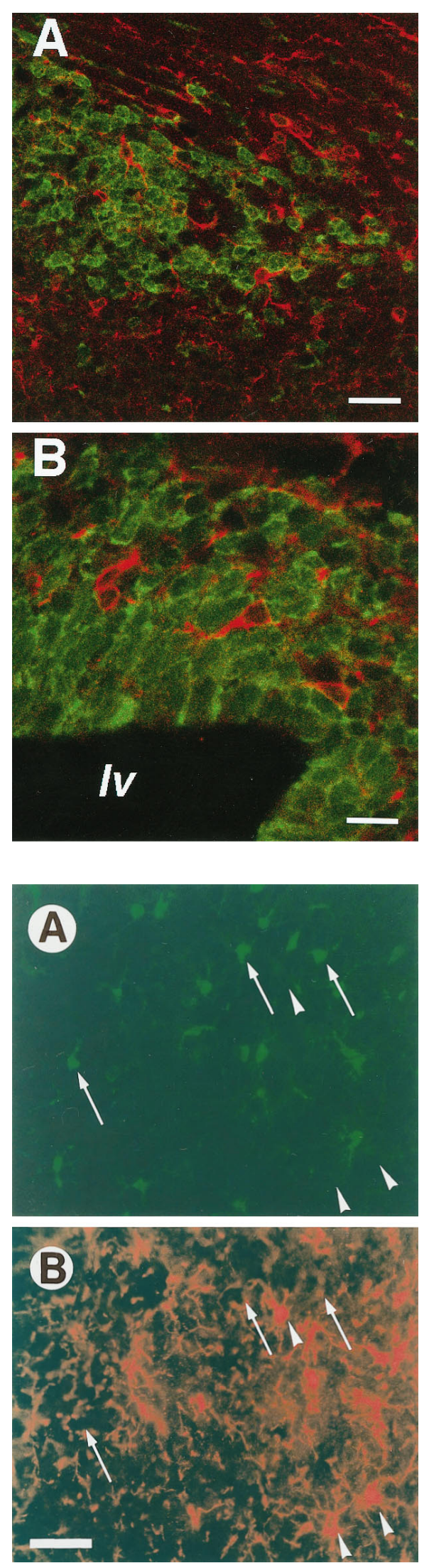

Figure 4. Top. Absence of m-Msi-1 expression in PDGF $\alpha$-R-positive, early O-2A cells lying in the SVZ. Scanning confocal images representing the expression of PDGF $\alpha-\mathrm{R}$ (red) and m-Msi-1 (green). A, Coronal section through the SVZ surrounding the dorsolateral corner of the lateral
1987; Fulton et al., 1992). Double-labeled sections of the P7 rat optic nerve showed that neither the $\operatorname{PDGF} \alpha$-R/NG2-positive $\mathrm{O}-2 \mathrm{~A}$ cells nor the CNPase-positive oligodendrocytes expressed $\mathrm{m}$-Msi-1, but there were a few astrocytes that expressed both m-Msi-1 and GFAP (data not shown).

\section{The gray matter}

In the developing cerebral cortex at P3, there were only a few cells immunoreactive for m-Msi-1 (data not shown). At P7, as the stratification of the cortex proceeded, we observed the accumulation of m-Msi-1-positive cells throughout the cerebral cortex (Fig. $6 C$ ), where most of them had small, round to oval cell bodies and a few short processes, resembling astrocytes in the process of differentiation. In contrast, $\mathrm{Hu}$ proteins were expressed exclusively in a population of large round neurons in the $\mathrm{P} 7$ cortical gray matter, distinct from the m-Msi-1-positive small cells (Fig. 6D). At subsequent developmental stages into the adult, there was a continuously increasing number of m-Msi-1-positive cells. These cells were distributed uniformly within the cortex and had small cell bodies with a few or multiple long processes extending in all directions (Fig. $6 E, G-J$ ), giving them an appearance of differentiated astrocytes. Hu was still expressed in large round cells, which seemed to be neurons, throughout the forebrain, including the pyramidal neurons in the cerebral cortex (Fig. 6F). Doublelabeling with $\mathrm{Hu}$ and $\mathrm{m}-\mathrm{Msi}-1$ antibodies clearly revealed that cells that were intensely positive for m-Msi-1 were $\mathrm{Hu}$-negative, and conversely, cells with larger, round cell bodies that expressed high levels of $\mathrm{Hu}$ proteins were $\mathrm{m}$-Msi-1-negative, indicating that $\mathrm{m}$ Msi-1 was expressed in non-neuronal cells (data not shown). Moreover, many astrocytes closely associated with vascular blood vessels, often appearing to make contact with the endothelial cells (data not shown) and cells lying under the pial surface (Fig. $6 K$ ), were also immunoreactive for m-Msi-1. Most of the m-Msi-1-positive cells in the adult cortex seemed to express the antigen throughout the entire cell body and processes (Fig. $6 G-J$ ).

To confirm the glial cell types expressing m-Msi-1 in the adult cerebral cortex, double-label immunofluorescence experiments were performed. The distribution of m-Msi-1-positive cells was compared with that of cells stained by the glial markers CNPase and GFAP. Sections double-labeled with antibodies to m-Msi-1 and GFAP revealed that there were many cells immunoreactive for both antigens in the adult cerebral cortex, as expected (Fig. 6), although a proportion of cells expressing m-Msi-1 were not reactive for GFAP. Cells that were positive for both m-Msi-1 and GFAP, which were predominantly distributed in the molecular layer (layer I) and near the pial surface in the cerebral cortex,

$\leftarrow$

ventricle and the adjacent corpus callosum of the $\mathrm{P} 2$ mouse forebrain. $B$, Higher magnification of the SVZ surrounding the lateral ventricle. Many PDGF $\alpha$-R-positive cells, which seem to have small cell bodies with a few processes, are observed not only in the corpus callosum and the striatum but also in the SVZ, where m-Msi-1-positive small cells are condensed. Note that these two populations of cells distribute intermingled but never overlap with each other. Scale bars: $A, 20 \mu \mathrm{m} ; B, 10 \mu \mathrm{m}$. $l v$, Lateral ventricle.

Figure 5. Bottom. Absence of m-Msi-1 expression in NG2-positive O-2A cells. Double-labeling of a section through the $\mathrm{P} 7$ rat corpus callosum with anti-m-Msi-1 $(A)$ and anti-NG2 $(B)$ antibodies fails to reveal doublepositive cells. Arrows indicate m-Msi-1-positive and NG2-negative cells. Arrowheads point to cells that are m-Msi-1-negative but NG2-positive. Scale bar, $30 \mu \mathrm{m}$. 
Figure 6. Immunolocalization of $\mathrm{m}-\mathrm{Msi}-1$ and $\mathrm{Hu}$ in the developing and adult cerebrum. $A, B$, Serial sagittal sections showing the expression of m-Msi-1 $(A)$ and PCNA $(B)$ in the SVZ and the adjacent intermediate zone at P3. Dorsal is to the right. The lateral ventricle is marked with asterisks. At P3, m-Msi-1 is strongly expressed in tightly packed, small round cells in the SVZ, which seem to correspond to PCNA-positive proliferating cells. It is also noted that there are some m-Msi-1-positive cells with a few processes extending into the dorsal parenchyma as if they were migrating out of the SVZ. $C-F$, Serial sagittal sections of the P7 $(C, D)$ and adult $(E, F)$ cerebral cortex showing the distribution of m-Msi-1 $(C, E)$ and $\mathrm{Hu}$ antigens $(D, F)$. The pial surface is at the top. $G-J$, High-power photomicrographs of individual cells expressing $\mathrm{m}-\mathrm{Msi}-1$ in the gray matter (layers III-IV, $H$ and $I$ ) and white matter (layer I, $G$; subcortical white matter, $J$ ) in the adult cerebral cortex. By P7, in addition to the high level of expression of Msi-1 in the SVZ, small scattered m-Msi-1-positive cells become localized throughout the cortex. At the same time, $\mathrm{Hu}$ expression is almost confined to neurons residing in the cortical gray matter. In the adult cerebral cortex, a larger number of m-Msi-1-positive cells are distributed uniformly in both gray and white matter, and these have small oval cell bodies with multiple short processes $(G, I)$ or bipolar processes $(H$, $J)$. $\mathrm{Hu}$ antigens continue to be expressed in large round neurons in the gray matter. $M Z$, Marginal zone; $C P$, cortical plate; $C C$, corpus callosum; $S V Z$, subventricular zone. $I, I I$, $I I I, V$, and $V I$ represent the cortical layers with the same designation. $K-N$, Double-immunofluorescent labeling of sections through the adult cerebral cortex: m-Msi-1 $(K)$, GFAP $(L)$, m-Msi-1 $(M)$, and CNPase $(N)$. m-Msi-1positive cells, which have multiple branched processes, are seen throughout the cortex. Astrocytes in the superficial molecular layer and near the pial surface show colocalization of m-Msi-1 and GFAP (arrowheads in $K$ and $L$ ), whereas CNPase-positive oligodendroglial cell bodies predominantly present in the molecular layer are m-Msi-1negative (arrows in $M$ and $N$ ). Scale bars: A, B, $18 \mu \mathrm{m}$; C, $\mathrm{D}, 71 \mu \mathrm{m}$; E, F, $36 \mu \mathrm{m}$; G-J, $8 \mu \mathrm{m} ; \mathrm{J}-\mathrm{N}, 10 \mu \mathrm{m}$.
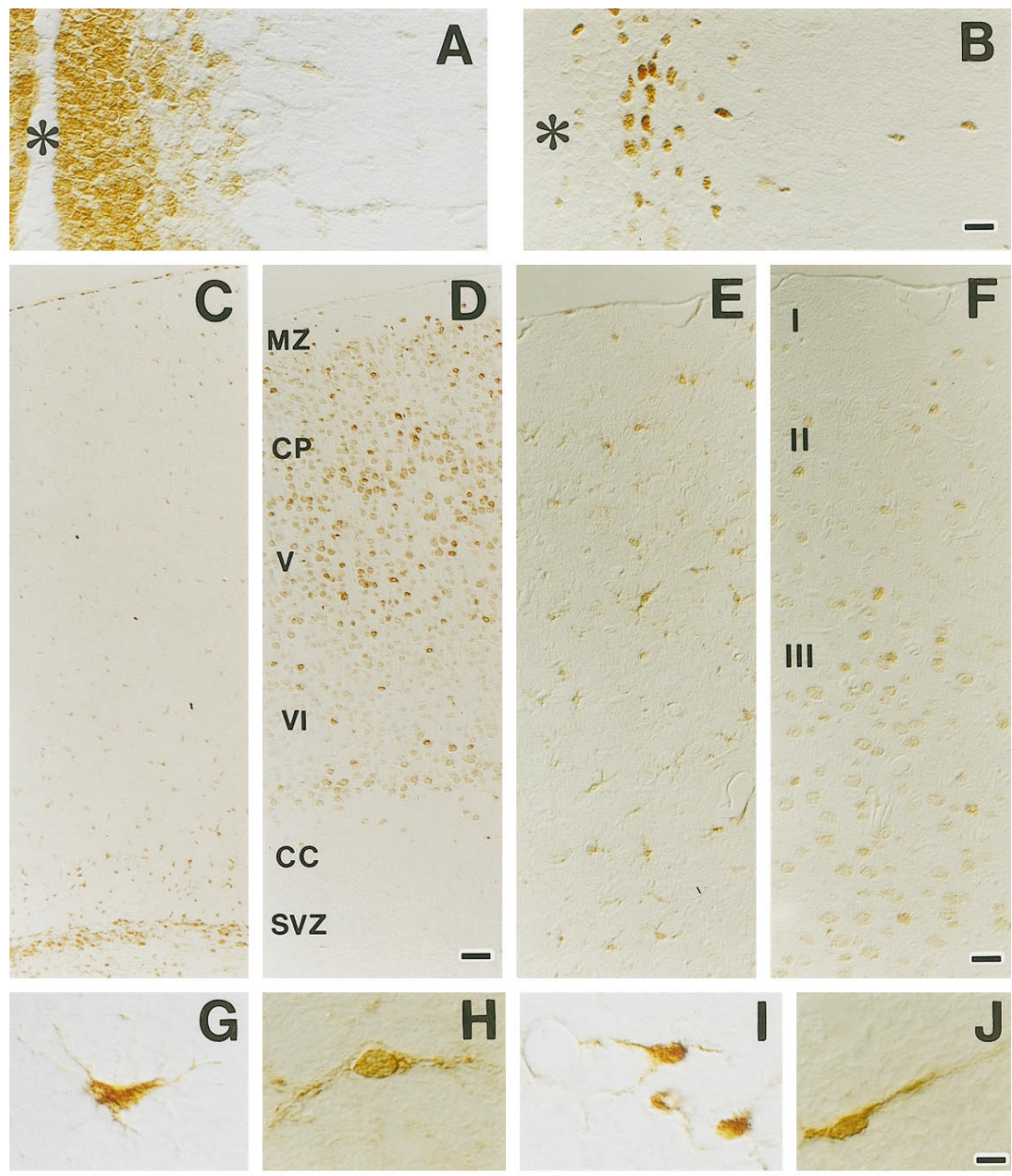

II
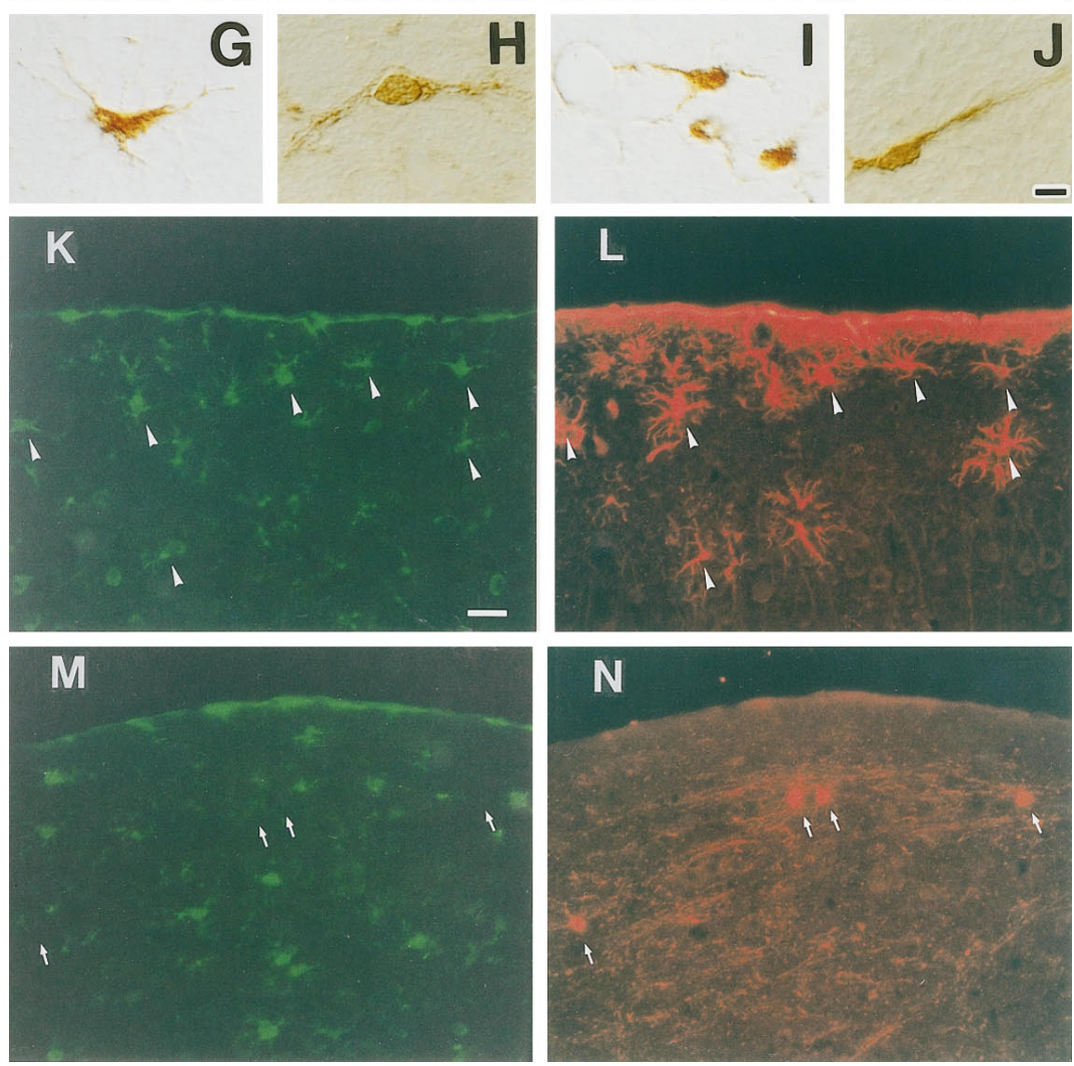

possessed relatively thick and branched processes, typical of differentiated astrocytes (Fig. 6). In addition, a considerable number of m-Msi-1-positive but GFAP-negative cells were observed in the deeper layers (layers II-VI) of the cortex (Fig. 6E,K,L). These cells may represent protoplasmic astrocytes, which are numerous in cortical layers II-VI but are sparse in layer I. Many of them were not stained by GFAP immunohistochemistry (Miller and Raff, 1984).

In oligodendrocytes, nonoverlapping populations of cells were positive for CNPase or m-Msi-1 in the adult cerebral cortex. 
Many CNPase-positive oligodendrocytes could be found in the molecular layer of the gray matter and the subcortical white matter of the cerebral cortex. These cells had elongated cell bodies and processes branching parallel to the axons (Fig. $6 N$ ). Although m-Msi-1-positive cells and CNPase-positive cells were found to be comingled throughout the gray and white matter, CNPase-positive cells were exclusively negative for m-Msi-1, and m-Msi-1-positive cells expressed no CNPase (Fig. 6M, $N$ ). Oligodendrocytes labeled with CNPase seemed to have oval cell bodies and processes that were finer than those of m-Msi-1positive cells.

These data indicated that m-Msi-1 expression was largely restricted to the mitotic glial precursor cells in the SVZ and astrocytes, which included GFAP-negative protoplasmic astrocytes and GFAP-positive differentiated astrocytes.

\section{m-Msi-1 expression in the cerebellum}

A dynamic pattern of m-Msi-1 expression was also observed in the developing cerebellum. In the cerebellar primordium at embryonic day 16.5, m-Msi-1 was largely expressed in the tightly packed small cells of the external granule cell layer (EGL) migrating out of the rhombic lip of the lateral recess, and also in the ventricular zone of the fourth ventricle (data not shown). By P3, significant m-Msi-1 expression could be detected in the EGL (Fig. $7 D$ ), which covers the surface of the developing cerebellum and is known to be composed exclusively of proliferating neuronal precursors. As neurogenesis progresses, two populations of cells appear in the EGL: cells proliferating in the superficial aspect of the EGL (EGLa) and cells undergoing the initial step of neuronal differentiation in the deeper aspect of the EGL (EGLb) (Altman, 1972; Kuhar et al., 1993). At P7, m-Msi-1 expression was mainly observed within the PCNA-positive proliferating cells in the superficial EGLa, although very low expression was also detected in the deeper layer EGLb (Fig. 7A,C). Conversely, Hu expression was restricted to the PCNA-negative postmitotic granule neurons within the EGLb (Fig. 7B,C). The numerous neurons in the internal granule cell layer (IGL), including the Purkinje cells forming a single row, were reactive for $\mathrm{Hu}$ but not for $\mathrm{m}-\mathrm{Msi}-1$ (Fig. $7 A, B$, arrowheads).

In addition, there were a large number of m-Msi-1-positive cells within the developing IGL, the deep cerebellar regions, and the putative white matter tracts (Fig. $7 A, D$ ). Most of these cells showed small oval cell bodies with unipolar or bipolar processes, which seemed to be migrating or differentiating glial cells. mMsi-1 was also strongly expressed in small round cells without processes in the SVZ lining the fourth ventricle, including the cerebral aqueduct, and in the superior medullary velum located at the base of the cerebellum (Fig. 7D), whereas $\mathrm{Hu}$ was completely absent from these cells (data not shown). Small numbers of scattered m-Msi-1-positive cells were observed over the developing inferior colliculus, which was continuous with the base of the cerebellum via the superior medullary velum, but the number was small compared with those seen in the cerebellum (Fig. 7D). Although we have not demonstrated the route of migration of m-Msi-1-positive cells per se, the expression pattern of m-Msi-1 in the developing cerebellum seemed to be consistent with patterns of glial precursor cells and migrating immature glial cells that originate from the SVZ cells of the fourth ventricle (Goldman et al., 1984; Curtis et al., 1988; Reynolds and Wilkin 1988).

In the adult there was a decrease in the number of m-Msi-1positive cells, although expression was still found in scattered cells in most regions of the cerebellum (Fig. 7G). In the Purkinje cell layer, which contains the Purkinje neurons and cell bodies of the Bergmann glia, m-Msi-1 expression seemed to be restricted to the radially aligned Bergmann glial cells and their fibers that course from the Purkinje cell layer through the molecular layer to the pial surface and was not detected in the adjacent Purkinje neurons (Fig. 7E, G,I). In the IGL, most of the m-Msi-1-positive cells were sparsely distributed, with small oval cell bodies and many short processes (Fig. 7E,J). The distribution and morphology were clearly distinct from those of $\mathrm{Hu}$-positive granule neurons (Fig. $7 B, F, H)$. In the central white matter tracts of the cerebellum, m-Msi-1 was expressed in scattered oval cells bearing unipolar or bipolar processes (Fig. $7 A, K$ ). To assess the type of cells that were immunoreactive for m-Msi-1, we performed indirect double-labeling on tissue sections of adult cerebellum with antibodies to GFAP, CNPase, and Hu. Colocalization of m-Msi-1 and GFAP was observed in various regions, including the Purkinje cell layer (Bergmann glia), IGL, and foliar white matter tracts. Neither CNPase nor Hu, however, was coexpressed with $\mathrm{m}-\mathrm{Msi}-1$ in these regions (data not shown), confirming that $\mathrm{m}$ Msi-1 was expressed in differentiated astrocytes, including Bergmann glial cells. However, we noticed that small populations of differentiated neurons in the cerebellar nuclei or interneurons, such as the stellate cells residing in the molecular layer, expressed m-Msi-1 (Fig. 7E-H). Similarly, we observed a weak expression of $\mathrm{m}-\mathrm{Msi}-1$ in some populations of differentiated neurons in the suprageniculate nucleus and paraventricular nucleus of the adult thalamus and hypothalamus (data not shown).

Taken together, the spatiotemporal distribution of m-Msi-1 strongly supports the idea that during the development of the cerebellum, the expression of m-Msi-1 is mainly restricted to the glial precursor cells and postmitotic and differentiated astrocytes, in addition to proliferating neuronal precursors found in the EGL. This expression pattern seemed to be comparable with that in the developing cerebrum. However, the expression of m-Msi-1 in some populations of differentiated neurons cannot be explained in this context.

\section{Changes in m-Msi-1 immunoreactivity after injury}

The present results indicating that m-Msi-1 was expressed in the cells of astrocyte lineage in postnatal brain led us to examine the changes in m-Msi-1 expression during the formation of glial scars after CNS injury. Small puncture lesions were made in the cerebral cortices of anesthetized adult mice. The appearance of normal adult cerebral tissue stained with the m-Msi- 1 antibody is shown in Figure $6 E$ as a control. Experimentally induced damage to the CNS leads to multiple changes in the glial cells surrounding the damaged tissue and to formation of a glial scar at the site of injury. Immunohistochemical and autoradiographic studies have indicated that hypertrophic and hyperplastic reactive astrocytes in the regions immediately adjacent to the injury site display increased levels of GFAP and become mitotically active (Miyake et al., 1988, 1992; Takamiya et al., 1988).

At $1 \mathrm{~d}$ postlesion, most of the m-Msi-1-positive cells around the lesion site exhibited no significant differences from m-Msi-1positive cells in undamaged areas of the same tissue section (data not shown). At $4 \mathrm{~d}$ postlesion, there was a dramatic increase in the number of m-Msi-1-positive cells in the vicinity of the injury site of the cerebral cortex (the region within 100-200 $\mu \mathrm{m}$ of the injury site) (Fig. 8). Compared with cells lying within the undamaged cerebral hemisphere, these cells exhibited an enhanced reactivity to m-Msi-1 antibody and had enlarged or elongated cell bodies with thick processes, occasionally with unipolar or bipolar pro- 
Figure 7. Distribution of $\mathrm{m}-\mathrm{Msi}-1$ and $\mathrm{Hu}$ in the developing $(A-D)$ and adult $(E-K)$ cerebellum. $D$, m-Msi-1 expression in the $\mathrm{P} 3$ cerebellum. m-Msi-1 is expressed in neuronal precursor cells in the external granule cell layer $(E G L)$ and in the vast number of small cells that have a few processes in the deep cerebellar regions. Intense m-Msi- 1 staining is also noted in densely packed small cells in the SVZ lining the fourth ventricle $(I V)$ and in the superior medullary velum $(S M V)$ located at the base of the cerebellum. $A-C$, Serial sagittal sections showing the immunolocalization of m-Msi-1 $(A), \mathrm{Hu}(B)$, and PCNA $(C)$ in the EGL, the deep cerebellar regions, and folia white matter $(W M)$ at $\mathrm{P} 7$. The EGL is toward the top of the panels. At P7, weak expression of m-Msi- 1 is seen mainly in the EGLa, which contains the PCNA-positive outer proliferating zone of the EGL, whereas $\mathrm{Hu}$ expression is observed in the $E G L b$, which contains the PCNA-negative inner early differentiating neurons. Granule neurons within the internal granule layer (IGL) and Purkinje cells (arrowheads) forming a single row show immunoreactivity for $\mathrm{Hu}$ but not for m-Msi-1. mMsi-1 expression is retained by small oval cells that have a few processes and reside in the deep cerebellar regions and WM. $G, H$, Sagittal sections showing the expression of m-Msi-1 $(G)$ and $\mathrm{Hu}(H)$ in the adult cerebellum. $E, F$, Higher magnifications of the molecular layer $(M L)$, Purkinje cell layer $(P L)$, and IGL shown in $G$ and $H$, respectively. $I-K$, High-power photomicrographs of individual cells expressing m-Msi- 1 in the PL $(I)$, IGL $(J)$, and WM $(K)$ of the adult cerebellum. In the PL, m-Msi-1 is expressed in Bergmann glia, the cell bodies of which are located adjacent to the large cell bodies of Purkinje cells $(P r)$ and extend their tangential processes into the ML. In addition, sparsely distributed putative astrocytes in the IGL and WM, which exhibit oval or elongated cell bodies with multiple short processes $(J)$ or bipolar processes $(K)$, are labeled with the m-Msi-1 antibody. $I C$, Inferior colliculus. Scale bars: $A-C, 36 \mu \mathrm{m} ; D, G, H, 71 \mu \mathrm{m} ; E, F, 25 \mu \mathrm{m}$; $I-K, 8 \mu \mathrm{m}$.
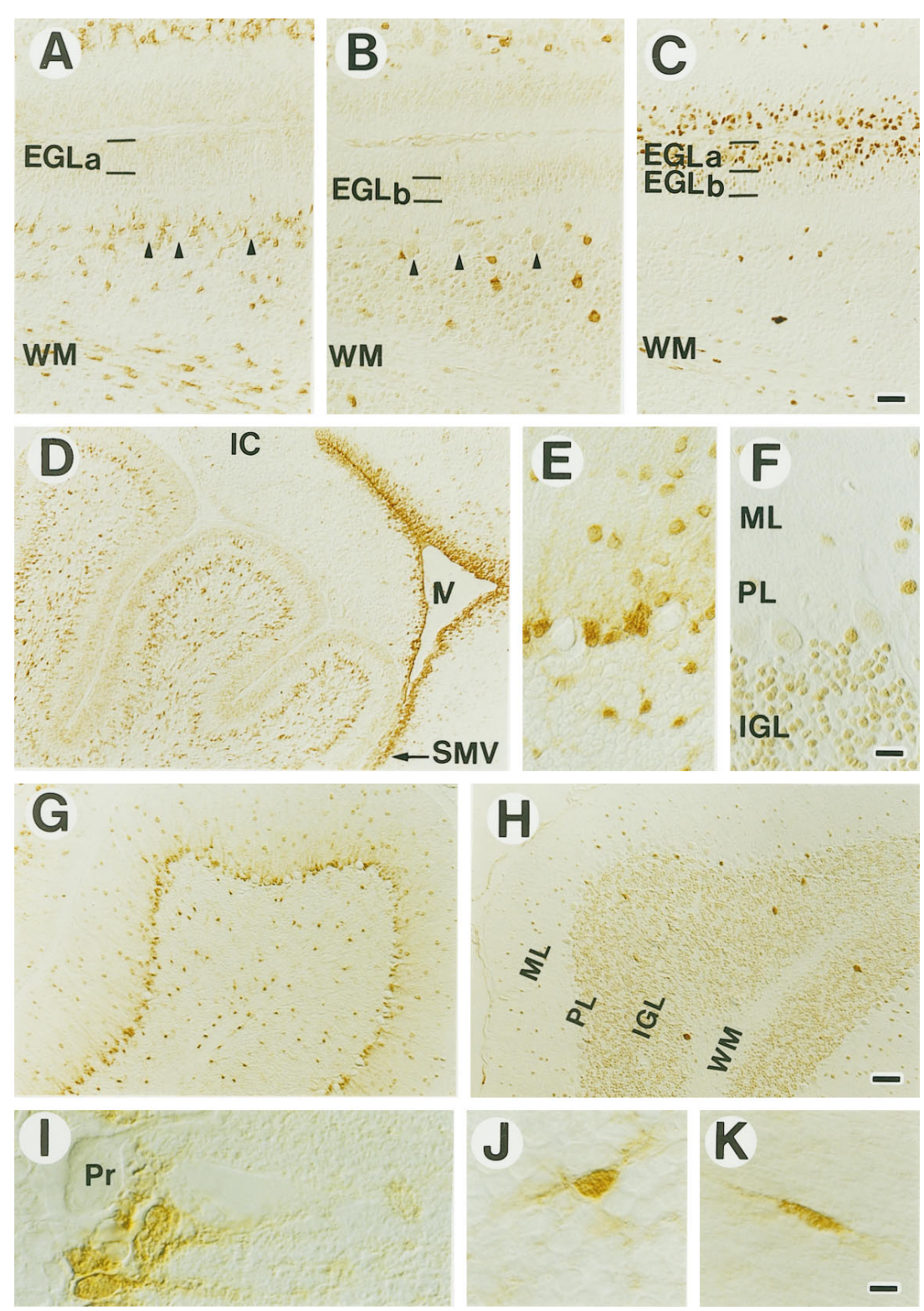

cesses oriented parallel to the needle track (Fig. 8). In addition to the effects in the immediate vicinity of the injury, there were also significant changes farther away from the injury site (regions $>200 \mu \mathrm{m}$ from the lesion; data not shown). The fact that these morphologically altered cells were not confined to the borders of the lesion suggests that the changes in m-Msi-1 expression are not attributable to direct physical damage of the cells.

To determine whether this increased population of m-Msi-1positive cells represents a so-called reactive astrocyte that proliferates in an injured area and participates in a glial scar formation at the site of injury (Miyake et al., 1988, 1992), we performed double-label immunostaining for m-Msi-1 and GFAP. As shown in Figure 8, in areas immediately adjacent to the injury site, numerous GFAP-positive astrocytes were present that had long and wavy processes extending in all directions or parallel to the needle track and seemed to form a thick bundle of glial array in the scar tissue, characteristics typical of reactive astrocytes. Double-labeled sections revealed that most of the m-Msi-1positive cells in these areas also expressed GFAP (Fig. 8C,D). This colocalization of m-Msi-1 and GFAP and the morphological characteristics of the cells strongly suggested that at least in the regions adjacent to the injury site, m-Msi-1 was expressed at high levels in reactive astrocytes that have been generated in response to injury and have a hyperplastic appearance.

The increases in the level of m-Msi-1 staining and in the number of cells expressing m-Msi- 1 within the $4 \mathrm{~d}$ after injury suggest that this population of m-Msi-1-positive glial cells may be stimulated to proliferate by the injury. To assess this possibility directly, animals were given two injections of BrdU, and cerebral coronal sections were prepared for double-immunolabeling with antibodies to m-Msi-1 and BrdU. At $1 \mathrm{~d}$ postlesion, there were no m-Msi-1-positive cells that incorporated BrdU throughout the injured cerebrum (data not shown). At $4 \mathrm{~d}$ postlesion, a number of cells that had incorporated BrdU appeared in regions adjacent to the site of the lesion. Approximately $20 \%$ of these BrdUlabeled cells also expressed m-Msi-1 (Fig. 8A,B). These BrdUand m-Msi-1-positive cells exhibited enlarged or elongated cell bodies (Fig. 8A,B, arrowheads), similar to GFAP-positive reactive astrocytes. This was consistent with previous studies showing the occurrence of cell division among GFAP-expressing astrocytes 
induced by brain injury (Miyake et al., 1988, 1992). In addition, the colocalization of BrdU and m-Msi-1 was observed in a few cells that were $>100 \mu \mathrm{m}$ away from the injury site. These cells frequently appeared in the subependymal region and the corpus callosum in the injured hemisphere, although by our method of BrdU administration, we found no BrdU-positive cells within the uninjured brain hemisphere (data not shown). Interestingly, both of these sources of dividing m-Msi-1-positive cells also expressed another intermediate filament protein, nestin (Fig. 8E,F), which is a well defined marker for neural precursors or stem cells during normal development of the CNS (Hockfield and McKay, 1985; Frederiksen and McKay, 1988; Lendahl et al., 1990; Zimmerman et al., 1994). Although the uninjured adult forebrain had weak nestin immunoreactivity, marked induction of nestin was observed $4 \mathrm{~d}$ after injury in both regions adjacent to the injury site (Fig. $8 F$ ) as well as in regions distant from the injury site, such as the subependyma and the overlying corpus callosum (data not shown). The colocalized expression of nestin and m-Msi-1 after injury may further imply that the expression of m-Msi-1 is maintained by cells that are in a precursor state and able to proliferate in response to injury.

On the other hand, the area adjacent to the injury site contained many BrdU-positive proliferating cells that were immunonegative for m-Msi-1 at $4 \mathrm{~d}$ postlesion (Fig. 8). Most of these cells seemed to be activated microglia, which proliferated in response to the injury, or proliferating macrophages and monocytes, which rapidly infiltrated into the injured parenchyma from the circulation when the injury also disrupted the blood-brain barrier. To examine whether m-Msi-1 expression was induced in the microglia and macrophages after injury, sections through the damaged area were double-stained with antibodies to m-Msi-1 and the monoclonal antibody MacI (Thomas, 1992), which identifies microglia, macrophages, and monocytes. As shown in Figure 8 , MacI-positive cells rapidly became activated by injury and increased in number in the damaged tissue. At $4 \mathrm{~d}$ postlesion, numerous MacI-positive cells appeared at the borders of the injury and formed a dense network, surrounding and partially filling the injury site (Fig. $8 H$ ). These cells seemed to be composed largely of activated ameboid microglia having large round cell bodies, and partially composed of macrophages and monocytes, which infiltrated from the circulation. There were also many MacI-positive ramified cells, located $50 \mu \mathrm{m}$ or more from the injury site, which were microglia and were unaffected by the injury (Fig. $8 H$, arrows). Indirect fluorescent double-labeling revealed that m-Msi-1 staining did not overlap with either the ameboid MacI-positive cells at the borders of the injury or the ramified ones in locations distant from the site of injury (Fig. $8 G, H)$. This observation showed that both activated and ramified microglia did not express m-Msi-1.

\section{DISCUSSION}

\section{m-Msi-1 may function in the generation of astrocytes in addition to neuronal precursor cells}

The present results indicated that $\mathrm{m}-\mathrm{Msi}-1$ is expressed in proliferative neuronal precursor cells, such as cells in the cerebellar EGLa and cells migrating from the SVZa into the olfactory bulb in the early postnatal CNS. Consistent with this, m-Msi-1-positive cells are present in the proliferative ventricular zone of the embryonic forebrain and spinal cord (Sakakibara et al., 1996). In addition, we showed that m-Msi-1 is expressed in proliferative glial precursor cells present in the postnatal SVZ and in constitutively proliferating cells (neural stem cells) in the adult subependyma, which may differentiate into neurons and glia in vitro (Lois and Alvarez-Buylla, 1993; Gritti et al., 1996; Johe et al., 1996).

$\mathrm{m}-\mathrm{Msi}-1$ is likely to be preferentially expressed in the astrocyte lineage during the development of glial cells. This does not necessarily suggest, however, that m-Msi-1-positive precursor cells in the SVZ have fates that are more restricted toward astrocyte lineage in glial cell development. Because a recent study using retroviral labeling of a single SVZ precursor cell in vivo in postnatal rats demonstrated the presence of bipotential glial precursor cells that give rise to both oligodendrocytes and astrocytes (Levison and Goldman, 1993), m-Msi-1-positive precursor cells in the SVZ may also potentially give rise to oligodendrocytes. However, m-Msi-1 was not expressed in the PDGF $\alpha$-R/NG2positive $\mathrm{O}-2 \mathrm{~A}$ cells, the probable intermediate precursors of the oligodendrocyte lineage (Ellison and de Vellis, 1994; Nishiyama et al., 1996). Thus, if m-Msi-1 is expressed in the bipotential glial precursor cells in the SVZ, m-Msi-1 expression must be largely downregulated at the very early stage of differentiation of oligodendrocyte lineage when $\operatorname{PDGF} \alpha-\mathrm{R} / \mathrm{NG} 2$ expression begins in O-2A cells (Fig. 9).

The role of $\mathrm{m}-\mathrm{Msi}-1$ in the generation of astrocytes from precursor cells in the SVZ during the early postnatal development of the forebrain may be explained by the following sequential events. First, proliferating m-Msi-1-positive glial precursors in the SVZ give rise to numerous m-Msi-1-positive and GFAPnegative immature astrocytes exhibiting single or bipolar processes and predominantly residing in the regions adjacent to the SVZ. Subsequently, these immature astrocytes migrate into the overlying parenchyma dorsal and lateral to the corpus callosum, and finally a population of these cells become dispersed in almost all regions of the forebrain where they have terminally differentiated and undergone morphological changes or acquired GFAP immunoreactivity. This developmental profile of astrocytes from SVZ precursor cells is also supported by previous ${ }^{3} \mathrm{H}$-thymidine labeling studies (Paterson et al., 1973; Imamoto et al., 1978) and lineage tracing of retroviral-labeled SVZ precursor cells (Levison and Goldman, 1993; Levison et al., 1993; Zerlin et al., 1995).

What type of astrocytes are m-Msi-1-positive cells? Astrocytes in the adult cerebrum are classified into two major types based on morphology and anatomical distribution. GFAP-positive fibrous astrocytes are located mainly in the white matter and the molecular layer of the cortex, and protoplasmic astrocytes are found mainly in the gray matter and frequently exhibit undetectable levels of GFAP-immunoreactivity (Miller and Raff, 1984). In the adult cerebrum, coexpression of m-Msi-1 and GFAP was observed predominantly in the superficial molecular layer, whereas there were a large number of m-Msi-1-positive but GFAPnegative cells in the deeper gray and white matter. Thus, the majority of m-Msi-1-positive astrocytes in the molecular layer and in deeper layers of cortex may correspond to the anatomically defined fibrous and protoplasmic astrocytes, respectively. In this context, m-Msi-1 may provide an excellent marker for astrocytes, including GFAP-negative protoplasmic astrocytes.

After brain injury, upregulation of m-Msi-1 expression was observed in a vast number of cells in broad regions of the injured cerebral hemisphere, including the subependyma distant from the injury site, as well as in the GFAP-positive proliferating reactive astrocytes in the immediate vicinity of the lesioned site. Previous studies suggested that protoplasmic astrocytes can transform into the fibrous type in response to injury and that the marked increase in the number of GFAP-positive reactive astrocytes in the injured cortex is principally attributable to upregulation of GFAP 

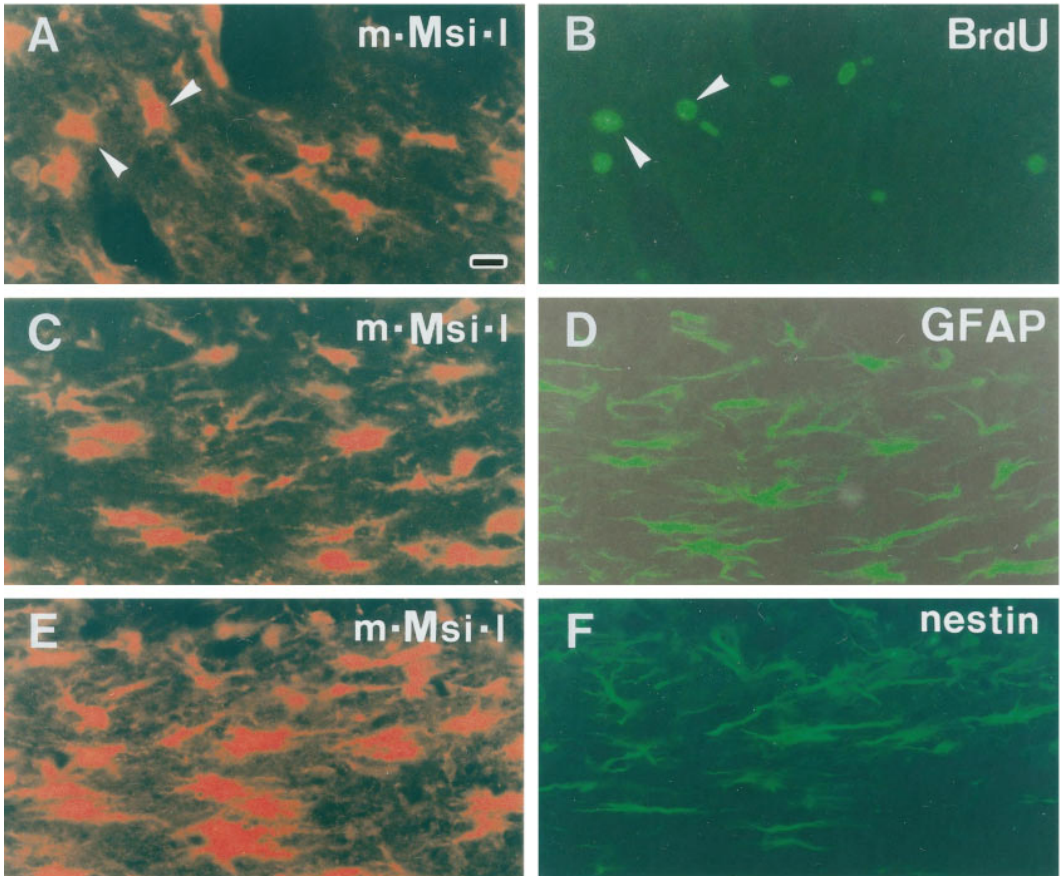

Figure 8. m-Msi-1 expression in reactive astrocytes in the injured region of adult cerebral cortex. Double-label fluorescent localization of m-Msi-1 and BrdU, GFAP, nestin, or MacI at $4 \mathrm{~d}$ postlesion. I, Schematic illustration of a coronal section through the injured forebrain. All photomicrographs correspond to the boxed region in $I$. The lesioned sites are toward the top in all panels. $A, \mathrm{~m}-\mathrm{Msi}-1$ (Cy3). $B$, Same field, BrdU (FITC). To label a proliferating population after brain injury, mice received BrdU injections for 3 $\mathrm{hr}$ before they were killed. m-Msi- 1 is expressed in a population of BrdU-positive proliferating cells (arrowheads) that lie close to the injury site and have enlarged cell bodies with multiple processes. $C$, m-Msi-1 (Cy3). D, Same field, GFAP (FITC). Intense m-Msi-1 staining is observed in an increased number of GFAP-positive reactive astrocytes surrounding the lesioned site; these cells exhibit enlarged, elongated cell bodies with multiple processes. $E$, m-Msi- 1 (Cy3). F, Same field, nestin (FITC). The simultaneous expression of nestin is induced in a subpopulation of $\mathrm{m}$ Msi-1-positive reactive cells near the lesioned site. $G, \mathrm{~m}-$ Msi-1 (FITC). $H$, Same field, MacI (Cy3). Large numbers of macrophages or ameboid microglia, which are MacIpositive but m-Msi-1-negative, form a dense plaque surrounding the lesion site. Arrows indicate the MacI-positive ramified microglia that are immunonegative for m-Msi-1. Scale bar (shown in $A$ ): $18 \mu \mathrm{m}$. $l v$, Lateral ventricle; $c c$, corpus callosum; str, striatum.
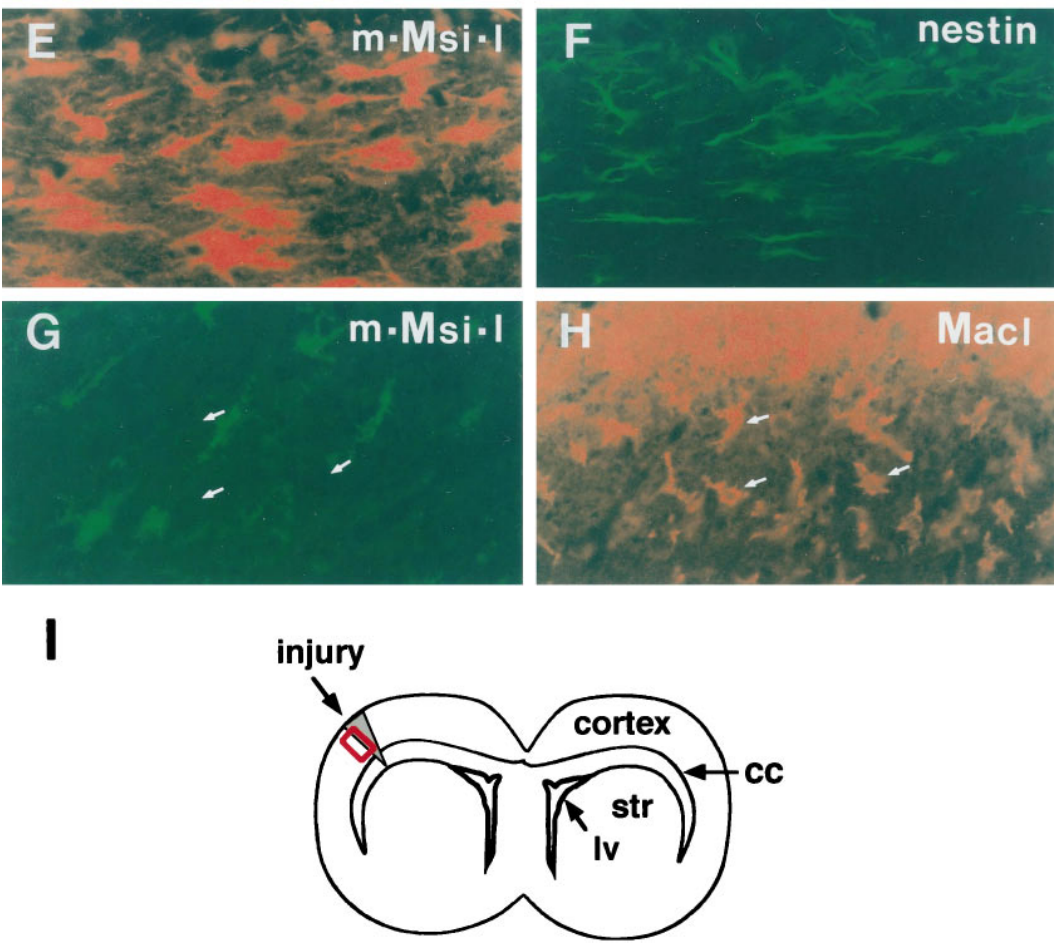

expression in already existing protoplasmic astrocytes surrounding the injury site, rather than to the induction of proliferation of GFAP-positive reactive astrocytes themselves (Bignami and Dahl, 1976; Graeber and Kreutzberg, 1986; Miyake et al., 1988, 1992). Consistent with the presumptive m-Msi-1 expression in the protoplasmic astrocytes in the normal cerebral cortex, the majority of m-Msi-1-positive cells observed at least in the vicinity of the lesioned site may represent overproduced protoplasmic astrocytes that are destined to become reactive astrocytes in the injured area. To confirm this possibility, however, lineage-tracing experiments of m-Msi-1-expressing cells are required (discussed below).

These results, taken together with the previous lineage study of SVZ cells using retroviral gene transfer (Levison and Goldman, 1993), suggest that m-Msi-1-positive cells in both the deeper cortical layer (GFAP-negative) and the molecular layer (GFAPpositive) may belong to the same astrocyte lineage derived from a common precursor cell proliferating in the SVZ. Then cells expressing m-Msi-1 but not GFAP may represent astrocytes that are in the process of development, before terminal differentiation.
To address these issues as well as the possible expression of m-Msi-1 in the bipotential glial precursor cells, it will be necessary to perform lineage-tracing experiments, for example using the GFP reporter driven by the $m-m s i-1$ promoter in a recombinant retrovirus, or knock-in mice combined with explant culture techniques. More sophisticated lineage experiments using the Cre/loxP system are also going to be examined.

\section{Possible functions of $\mathbf{m}$-Msi-1 in neural development}

On the basis of an analogy with Drosophila d-Msi, and the in vivo and in vitro expression pattern of m-Msi-1 in the embryonic CNS, we proposed previously that m-Msi-1 may be required for neuronal lineage formation from undifferentiated neural precursor cells that can give rise to both neurons and glia, or in the maintenance of these precursor cells (Sakakibara et al., 1996). In the present study, we observed m-Msi-1 expression not only in neuronal precursors but also in glial precursor cells and astrocytes during postnatal CNS development. m-Msi-1 protein may regulate the expression of RNA molecules, which are required for the maintenance of both neuronal and glial precursor cells and dif- 


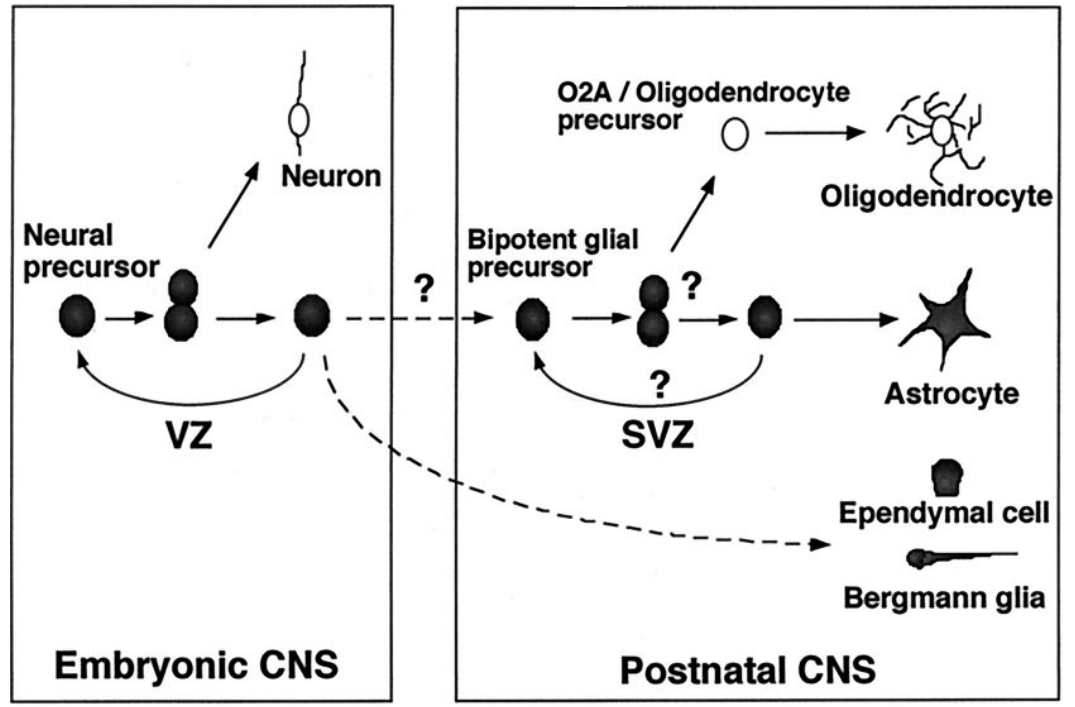

Figure 9. Schematic representation of cells expressing mMsi-1 during embryonic and postnatal CNS development. Solid patterns represent m-Msi-1-positive cells. In the embryonic CNS, m-Msi-1 is expressed in proliferating neural precursors in the ventricular zone $(V Z)$; these cells are capable of self-renewal and generate postmitotic neurons, as described previously (Sakakibara et al., 1996). In the perinatal stage, the VZ shrinks and a second proliferative SVZ appears. Postnatally, m-Msi-1 expression is seen in proliferating glial precursors in the SVZ and cells of astrocyte lineage, including ependymal cells and Bergmann glia. At present, it is not known whether neural precursors in the embryonic VZ generate glial precursors in the postnatal SVZ, or whether bipotent glial precursors in the postnatal SVZ generate the astrocyte and O-2A-oligodendrocyte lineages through an "asymmetric cell division." However, this asymmetric division at least may occur when neuronal precursors give rise to neurons in the embryonic VZ (Chenn and McConnell, 1995). The expression of m-Msi-1 in some populations of neurons is not represented for reasons of simplicity. ferentiated astrocytes. If m-Msi-1 was shown to be expressed in bipotent glial precursor cells that give rise to both O-2A/oligodendrocytes and astrocytes by the lineage studies, it would be tempting to speculate that m-Msi-1 might be involved in the determination and maintenance of an astrocyte lineage from a bipotent glial precursor cell in SVZ (summarized in Fig. 9). This may be achieved by controlling the expression of target genes to repress the generation of $\mathrm{O}-2 \mathrm{~A} /$ oligodendrocyte lineage from a bipotent glial precursor cell, consistent with the observations showing the absence of $\mathrm{m}-\mathrm{Msi}-1$ expression from the early stage of oligodendrocyte (O-2A) development and persistent expression in the SVZ glial precursor cells. This idea is also comparable with the loss of m-Msi-1 expression in differentiating neurons during embryonic (Sakakibara et al., 1996) and postnatal neurogenesis (Fig. 9). Thus, m-Msi-1 may regulate common key RNA molecules, which are required for the determination of these cell-fates from both glial and neuronal precursors.

The elucidation of the target RNA molecules of m-Msi-1, which is currently in progress in our laboratory, will unequivocally reveal the function of this protein. Phenotypic analysis of $m-m s i-1$ knock-out mice will also help us to understand the role of this gene product in the embryonic and postnatal CNS development.

\section{REFERENCES}

Altman J (1972) Postnatal development of the cerebellar cortex in the rat. J Comp Neurol 145:353-398.

Bignami A, Dahl D (1974) Astrocyte-specific protein and neuroglial differentiation. An immunofluorescence study with antibodies to the glial fibrillary acidic protein. J Comp Neurol 153:27-38.

Bignami A, Dahl D (1976) The astroglial response to stabbing. Immunofluorescence studies with antibodies to astrocyte-specific protein (GFA) in mammalian and submammalian vertebrates. Neuropathol Appl Neurobiol 2:99-110.

Chenn A, McConnell SK (1995) Cleavage orientation and the asymmetric inheritance of Notch1 immunoreactivity in mammalian neurogenesis. Cell 82:631-641.

Craig CG, Tropepe V, Morshead CM, Reynolds BA, Weiss S, van der Kooy D (1996) In vivo growth factor expansion of endogenous subependymal neural precursor cell populations in the adult mouse brain. J Neurosci 16:2649-2658.

Curtis R, Cohen J, Fok-Seang J, Hanley MR, Gregson NA, Reynolds R, Wilkin GP (1988) Development of macroglial cells in rat cerebellum. I. Use of antibodies to follow early in vivo development and migration of oligodendrocytes. J Neurocytol 17:43-54.

Davis AA, Temple S (1994) A self-renewing multipotential stem cell in embryonic rat cerebral cortex. Nature 372:263-266.
Ellison J, de Vellis J (1994) Platelet derived growth factor receptor is expressed by cells in the early oligodendrocyte lineage. J Neurosci Res $37: 116-128$.

Frederiksen K, McKay RDG (1988) Proliferation and differentiation of rat neuroepithelial precursor cells in vivo. J Neurosci 8:1144-1151.

Fulton BP, Burne JJ, Raff MC (1992) Visualization of O-2A progenitor cells in developing and adult rat optic nerve by quisqualate- stimulated cobalt uptake. J Neurosci 12:4816-4833.

Galand P, Degraef C (1989) Cyclin/PCNA immunostaining as an alternative to tritiated thymidine pulse labelling for marking $S$ phase cells in paraffin sections from animal and human tissues. Cell Tissue Kinet 22:383-392.

Gates MA, Thomas LB, Howard EM, Laywell ED, Sajin B, Faissner A, Götz B, Silver J, Steindler DA (1995) Cell and molecular analysis of the developing and adult mouse subventricular zone of the cerebral hemispheres. J Comp Neurol 361:249-266.

Goldman JE, Hirano M, Yu RK, Seyfried TN (1984) GD $_{3}$ ganglioside is a glycolipid characteristic of immature neuroectodermal cells. J Neuroimmunol 7:179-192.

Graeber MB, Kreutzberg GW (1986) Astrocytes increase in glial fibrillary acidic protein during retrograde changes of facial motor neurons. J Neurocytol 15:363-373.

Gritti A, Pareti EA, Cova L, Frolichsthal P, Galli R, Wanke E, Faravelli L, Morassutti DJ, Roisen F, Nickel DD, Vescovi AL (1996) Multipotential stem cells from the adult mouse brain proliferate and self-renew in response to basic fibroblast growth factor. J Neurosci 16:1091-1100.

Hardy R, Reynolds R (1991) Proliferation and differentiation potential of rat forebrain oligodendrocyte progenitors both in vitro and in vivo. Development 111:1061-1080.

Hockfield S, McKay R (1985) Identification of major cell classes in the developing mammalian nervous system. J Neurosci 5:3310-3328.

Imamoto K, Paterson JA, Leblond CP (1978) Radioautographic investigation of gliogenesis in the corpus callosum of young rats. I. Sequential changes in oligodendrocytes. J Comp Neurol 180:115-138.

Johe KK, Hazel TG, Muller T, Dugich-Djordjevic MM, McKay RDG (1996) Single factors direct the differentiation of stem cells from the fetal and adult central nervous system. Genes Dev 10:3129-3140.

Kuhar SG, Feng L, Vidan S, Ross ME, Hatten ME, Heinz N (1993) Changing patterns of gene expression define four stages of cerebellar granule neuron differentiation. Development 117:97-104.

Lendahl U, Zimmerman LB, McKay R (1990) CNS stem cells express a new class of intermediate filament protein. Cell 60:585-595.

Levine TD, Gao F, King PH, Andrews LG, Keene JD (1993) Hel-N1: an autoimmune RNA-binding protein with specificity for $3^{\prime}$ uridylate-rich untranslated regions of growth factor mRNAs. Mol Cell Biol 13:3494-3504.

Levison SW, Goldman JE (1993) Both oligodendrocytes and astrocytes develop from progenitors in the subventricular zone of postnatal rat forebrain. Neuron 10:201-212.

Levison SW, Chuang C, Abramson B, Goldman JE (1993) The migrational patterns and developmental fates of glial precursors in 
the rat subventricular zone are temporally regulated. Development 119:611-622.

Lewis PD (1968) A quantitative study of cell proliferation in the subependymal layer of the adult rat brain. Exp Neurol 20:203-207.

Liu J, Dalmau J, Szabo A, Rosenfeld M, Huber J, Furneaux H (1995) Paraneoplastic encephalomyelitis antigens bind to the AU-rich elements of mRNA. Neurology 45:544-550.

Lois C, Alvarez-Buylla A (1993) Proliferating subventricular cells in the adult mammalian forebrain can differentiate into neurons and glia. Proc Natl Acad Sci USA 90:2074-2077.

Lois C, Alvarez-Buylla A (1994) Long distance neuronal migration in the adult mammalian brain. Science 264:1145-1148.

Luskin MB (1993) Restricted proliferation and migration of postnatally generated neurons derived from the forebrain subventricular zone. Neuron 11:173-189.

Marusich MF, Furneaux HM, Henion PD, Weston JA (1994) Hu neuronal proteins are expressed in proliferating neurogenic cells. J Neurobiol 25:143-155.

Miller RH, Raff MC (1984) Fibrous and protoplasmic astrocytes are biochemically and developmentally distinct. J Neurosci 4:585-592.

Miller RH, David S, Patel R, Abney ER, Raff MC (1985) A quantitative immunohistochemical study of macroglial cell development in the rat optic nerve: in vivo evidence for two distinct astrocyte lineage. Dev Biol 111:35-41.

Miyata T, Ogawa M (1994) Developmental potentials of early telencephalic neuroepithelial cells: a study with microexplant culture. Dev Growth Differ 36:319-331.

Miyake T, Hattori T, Fukuda M, Kitamura T, Fujita S (1988) Quantitative studies on proliferative changes of reactive astrocytes in mouse cerebral cortex. Brain Res 451:133-138.

Miyake T, Okada M, Kitamura T (1992) Reactive proliferation of astrocytes studied by immunohistochemistry for proliferating cell nuclear antigen. Brain Res 590:300-302.

Morshead CM, van der Kooy D (1992) Postmitotic death is the fate of constitutively proliferating cells in the subependymal layer of the adult mouse brain. J Neurosci 12:249-256.

Morshead CM, Reynolds BA, Craig CG, McBurney MW, Staines WA, Morassutti D, Weiss S, van der Kooy D (1994) Neural stem cells in the adult mammalian forebrain: a relatively quiescent subpopulation of subependymal cells. Neuron 13:1071-1082.

Nakamura M, Okano H, Blendy J, Montell C (1994) Musashi, a neural RNA-binding protein required for Drosophila adult external sensory organ development. Neuron 13:67-81.

Nishiyama A, Lin XH, Giese N, Helden CH, Stallcup WB (1996) Colocalization of NG2 proteoglycan and PDGF a-receptor on O2A progenitor cells in the developing rat brain. J Neurosci Res 43:299-314.

Okano H (1995) Two major mechanisms regulating cell-fate decisions in the developing nervous system. Dev Growth Differ 37:619-629.

Okano HJ, Darnell RB (1997) A hierarchy of Hu RNA binding proteins in developing and adult neurons. J Neurosci 17:3024-3037.

Paterson JA, Privat A, Ling EA, Leblond CP (1973) Investigation of glial cells in semithin sections. III. Transformations of subependymal cells into glial cells as shown by autoradiography after $3 \mathrm{H}$-thymidine injection into the lateral ventricle of the brain of young rats. J Comp Neurol 149:83-102.

Pringle NP, Mudhar HS, Collarini EL, Richardson WD (1992) PDGF receptors in the rat CNS: during late neurogenesis, PDGF alphareceptor expression appears to be restricted to glial cells of the oligodendrocyte lineage. Development 115:535-551.

Privat A (1975) Postnatal gliogenesis in the mammalian brain. Int Rev Cytol 40:281-323.

Privat A, Leblond CP (1972) The subependymal layer and neighbouring region in the brain of the young rat. J Comp Neurol 146:277-302.

Reynolds R, Wilkin GP (1988) Development of macroglial cells in rat cerebellum II. An in situ immunohistochemical study of oligodendroglial lineage from precursor to mature myelinating cell. Development 102:409-425.

Richter K, Good PJ, Dawid IB (1990) A developmentally regulated, nervous system-specific gene in Xenopus encodes a putative RNAbinding protein. New Biologist 2:556-565.

Sakakibara S, Imai T, Hamaguchi K, Okabe M, Aruga J, Nakajima K, Yasutomi Y, Nagata T, Kurihara Y, Uesugi S, Miyata T, Ogawa M, Mikoshiba K, Okano H (1996) Mouse-Musashi-1, a neural RNAbinding protein highly enriched in the mammalian CNS stem cell. Dev Biol 176:230-242.

Small RK, Riddle P, Noble M (1987) Evidence for migration of oligodendrocyte-type-2 astrocyte progenitor cells into the developing rat optic nerve. Nature 328:155-157.

Smart I (1961) The subependymal layer of the mouse brain and its cell production as shown by radioautography after thymidine- $\mathrm{H}^{3}$ injection. J Comp Neurol 116:325-338.

Sprinkle TJ (1989) 2', 3'-Cyclic nucleotide 3'-phosphodiesterase, an oligodendrocyte-Schwann cell and myelin-associated enzyme of the nervous system. CRC Crit Rev Neurobiol 4:235-301.

Stallcup WB, Beasley L (1987) Bipotential glial precursor cells of the optic nerve express the NG2 proteoglycan. J Neurosci 7:2737-2744.

Szabo A, Dalmau J, Manley G, Rosenfeld M, Wong E, Henson J, Posner JB, Furneaux HM (1991) Hu, a paraneoplastic encephalomyelitis antigen, contains RNA-binding domains and is homologous to Elav and Sex-lethal. Cell 67:325-333.

Takakura N, Yoshida H, Kunisada T, Nishikawa S, Nishikawa SI (1996) Involvement of platelet-derived growth factor receptor-alpha in hair canal formation. J Invest Dermatol 107:770-777.

Takamiya Y, Kohsaka S, Toya S, Otani M, Tsukada Y (1988) Immunohistochemical studies on the proliferation of reactive astrocytes and the expression of cytoskeletal proteins following brain injury in rats. Dev Brain Res 38:201-210.

Thomas WE (1992) Brain macrophages: evaluation of microglia and their functions. Brain Res Rev 17:61-74.

Yao K-M, Samson M-L, Reeves R, White K (1993) Gene elav of Drosophila melanogaster: a prototype for neuron-specific RNA binding protein gene family that is conserved in flies and humans. J Neurobiol 24:723-739.

Zerlin M, Levison SW, Goldman JE (1995) Early patterns of migration, morphologenesis, and intermediate filament expression of subventricular zone cells in the postnatal rat forebrain. J Neurosci 15:7238-7249.

Zimmerman L, Lendahl U, Cunningham M, McKay R, Parr B, Gavin B, Mann J, Vassileva G, McMahon A (1994) Independent regulatory elements in the nestin gene direct transgene expression to neural stem cells or muscle precursors. Neuron 12:11-24. 